\title{
ANATOMÍA FOLIAR DE HIERBAS TERRESTRES MEDICINALES QUE CRECEN EN LA REGIÓN RIOPLATENSE (BUENOS AIRES, ARGENTINA)
}

\author{
MARCELO P. HERNÁNDEZ, MARÍA C. NOVOA, MARTA N. COLARES, VANESA G. PERROTTA, LEANDRO M. \\ NUGHES \& ANA M. ARAMBARRI ${ }^{1}$
}

\begin{abstract}
Summary: Hernández, M. P., M. C. Novoa, M. N. Colares, V. G. Perrotta, L. M. Nughes \& A. M. Arambarri. 2015. Foliar anatomy of medicinal land herbs growing in the Rio de La Plata region (Buenos Aires, Argentina). Bonplandia 24(2): 97-123.
\end{abstract}

This work is part of the project "Anatomy of the vegetative organs with medicinal properties in rioplatenses Spermatophytes. I. Land herbs". We studied leaf micrographic characteristics of 62 species belonging to 24 families. The aim was to create a dichotomous key to identify the taxa based on these leaf characteristics from both whole and fragmented plant material, and to provide a comparative table with information on quantitative parameters, important for the control of pharmacognostic quality. Mature leaves from living and herbarium specimens were studied using conventional histological techniques (peeling, epidermis print, diaphanization, cross sections). Starch, mucilage, lipophilic substances and mirosine were identified using microchemical methods. The principal differences were found in epidermal cells, distribution and stomata type, trichome types, leaf mesophyll, midrib characteristics, and stomatal and pilosity indexes. The presence of a conspicuous endodermis and the different types of crystals and internal secretory reservoirs (cells, cavities, and ducts) contributed to develop an identification key.

Key words: Anatomy, leaves, medicinal plants, Río de La Plata area.

Resumen: Hernández, M. P., M. C. Novoa, M. N. Colares, V. G. Perrotta, L. M. Nughes \& A. M. Arambarri. 2015. Anatomía foliar de hierbas terrestres medicinales que crecen en la región rioplatense (Buenos Aires, Argentina). Bonplandia 24(2): 97-123.

El presente trabajo se desarrolló en el marco del proyecto "Anatomía de los órganos vegetativos con propiedades medicinales de Espermatófitas rioplatenses. I. Hierbas terrestres". En esta oportunidad realizamos el estudio de las características micrográficas foliares de 62 especies pertenecientes a 24 familias. El objetivo fue generar una clave dicotómica para identificar los taxones a partir de las hojas, ya sea que este material se encuentre al estado entero o fragmentado y brindar información en un cuadro comparativo de los parámetros cuantitativos, los cuales son importantes herramientas en el control de calidad farmacognóstica. Se estudiaron hojas maduras frescas y de ejemplares de herbario

\footnotetext{
${ }^{1}$ Docentes de Morfología Vegetal y Sistemática Vegetal e Investigadores del Laboratorio de Morfología Comparada de Espermatófitas (LAMCE), Facultad de Ciencias Agrarias y Forestales, UNLP, 60 y 119, CC 31, $1900 \mathrm{La}$ Plata, Argentina. Tel. +54-221-423-6758, int. 461; Fax: +54-221-425-2346. Autor para correspondencia: E-mail: anaramba@yahoo.com.ar; botgral@agro.unlp.edu.ar
} 


\begin{abstract}
empleando técnicas histológicas convencionales ("peeling”, impronta, diafanización, cortes transversales). Se practicaron métodos microquímicos para la identificación de almidón, mucílagos, sustancias lipofílicas y mirosina. Las principales diferencias se encontraron en la forma de las células epidérmicas, distribución y tipo de estomas, tipos de tricomas, mesofilo foliar, características de la vena media e índices estomático y de pilosidad. La presencia de una endodermis conspicua; los diferentes tipos de cristales y reservorios secretores internos (células, cavidades y conductos) contribuyeron a la elaboración de una clave de diferenciación.
\end{abstract}

Palabras clave: Anatomía, hoja, plantas medicinales, región del Río de La Plata.

\section{Introducción}

Dentro de la Provincia Biogeográfica Pampeana con el aporte de las Provincias del Espinal y Paranaense se ubica la región rioplatense. Esta es una extensa área que se extiende desde Diamante en la provincia de Entre Ríos (324'0"S 60³9'0”O) hasta Punta Lara en la provincia de Buenos Aires (3447'28' S 5759'49''O). Este sector se divide en Delta superior, medio e inferior. La vegetación estudiada habita el sector inferior del Delta del Paraná, isla Martín García y ribera platense (Lahitte et al., 1997). Como se citó en Arambarri et al. (2013), existen numerosos trabajos desde el punto de vista florístico y sistemático de las plantas de esta zona (e. g., Hauman, 1925; Burkart, 1957; Cabrera, 1949, 1963; Cabrera \& Dawson, 1944; Cabrera \& Burkart, 1974; Cabrera \& Zardini, 1978). Numerosos autores han señalado las propiedades medicinales de las plantas que crecen en la región (Hieronymus, 1882; Domínguez, 1928; Toursarkissian, 1980; Lahitte \& Hurrell, 1996; Lahitte et al., 2004; Alonso \& Desmarchelier, 2005; Rondina et al., 2008, 2010; Barboza et al., 2006, 2009). Este trabajo es continuación del proyecto mencionado, en cuyo marco ya se realizaron las siguientes contribuciones sobre anatomía comparada e histoquímica: los tallos y rizomas de siete Monocotiledóneas (Novoa et al., 2012); los tallos aéreos de 37 Dicotiledóneas (Arambarri et al., 2013) y las raíces de 22 hierbas terrestres (Colares et al., 2014). En esta oportunidad estudiamos la anatomía e histoquímica de las hojas de 62 especies pertenecientes a 24 familias. Destacamos que existen numerosos trabajos sobre características anatómicas foliares (e.g., Bonzani et al., 1997; Gattuso \& Gattuso 1998; Barboza et al. 2001; Monti et al., 2003; Duarte \& Debur, 2004; Apóstolo, 2005; Cambi et al., 2006; Yagueddú et al., 2009; Stiefkens et al., 2010). Sin embargo, al presente estudio se le dió un enfoque comparativo de las características foliares de las especies relevadas como empleadas en la medicina tradicional de esta región (Lahitte \& Hurrell, 1996; Lahitte et al., 1997, 2004; Pochettino et al., 1997; Hernández et al., 2009, 2010, 2013; Hurrell et al., 2011a, b). El objetivo es analizar caracteres cualitativos y parámetros cuantitativos de la hoja en las 62 hierbas terrestres. Sobre la base de los caracteres cualitativos elaborar una clave dicotómica para la identificación de material de hoja en su estado entero o fragmentado y ofrecer un cuadro comparativo de los parámetros cuantitativos que faciliten el control de calidad botánica.

\section{Materiales y Métodos}

\section{Material estudiado}

Para el estudio se emplearon hojas frescas y de herbario. Las plantas frescas, completas, recolectadas por los autores, fueron determinadas, herborizadas y depositadas en el herbario de la Facultad de Agronomía de La Plata (LPAG). También se examinó material de ejemplares del herbario de la Facultad de Ciencias Naturales y Museo de La Plata (LP). El material analizado es de Argentina, en su mayoría fueron recolectados en la provincia de Buenos Aires, pero también se han analizado materiales 
procedentes de las provincias de Entre Ríos, Formosa y Santa Fe, como se indica en las siguientes referencias: AMARANTHACEAE: Alternanthera philoxeroides (Mart.) Griseb. f. philoxeroides. Buenos Aires: Pdo. La Plata, City Bell, 12-III-1995, Bayón 178; La Plata, 14-X2011, Arambarri 361 (LPAG). A. pungens Kunth. Buenos Aires: Ciudad Autónoma, V-2012, Auguet s. n. (LPAG). Formosa: Laguna Yema, 17-V-2004, Bayón \& Moreno 778 (LPAG). Santa Fe: Dpto. Nueve de Julio, Tostado, 1-II1936, Job 1124 (LP). Amaranthus hybridus L. ssp. hybridus. Buenos Aires: Pdo. La Plata, La Plata, 27-XII-1972, Bayón 615 (LPAG); Pdo. La Plata, Tolosa, 11-IV-2004, Bayón 633 (LPAG). APOCYNACEAE: Vinca major L. Buenos Aires: Pdo. La Plata, Isla Martín García, 1975, Hurrell et al. 2374 (LP); Pdo. La Plata, City Bell, 7-X-2013, Hernández 122 (LPAG); La Plata, 5-X-2013, Arambarri \& Vizcaino 503 (LPAG). ASTERACEAE: Achyrocline satureioides (Lam.) DC. Buenos Aires: Pdo. La Plata, Isla Martín García, 28-III-1997, Hurrell 3543 (LP); Pdo. de la Costa, San Clemente del Tuyú, s.f., Cabrera 4263 (LP). Acmella decumbens (Sm.) R. K. Jansen. Buenos Aires: Pdo. Berisso, Berisso, 8-X-2001, Torres \& Simon 512 (LP); Pdo. Ensenada, Punta Lara, 23-XI-2002, Torres \& De Gregorio 1028 (LP). Ambrosia tenuifolia Spreng. Buenos Aires: Pdo. Ensenada, Isla Santiago, 14-III-2012, Hernández 108 (LPAG). Bidens pilosa L. Buenos Aires: Pdo. La Plata, La Plata, 20-XI-2011, Hernández 87 (LPAG); 24IV-2012, Arambarri 379 (LPAG). Cichorium intybus L. Buenos Aires: Pdo. La Plata, Manuel B. Gonnet, s.f., Delucchi 1757 (LP). Conyza bonariensis (L.) Cronquist. Buenos Aires: Pdo. La Plata, La Plata, 5-I-2012, Hernández 96 (LPAG). Galinsoga parviflora Cav. Buenos Aires: Pdo. La Plata, Jardín Botánico y Arboretum (JBA “C. Spegazzini"), Facultad de Ciencias Agrarias y Forestales (FCAyF), Universidad Nacional de La Plata (UNLP), 26-II-2013, Arambarri 410 (LPAG). Gamochaeta coarctata (Willd.) Kerguélen. Buenos Aires: Pdo. La Plata, JBA “C. Spegazzini”, FCAyF, UNLP, 4-VI-2012, Arambarri 381 (LPAG); 16-VII-2012, Arambarri 382 (LPAG). Mikania micrantha Kunth. Buenos Aires: Pdo. Ensenada, Punta Lara, 10-XI-2008, Colares 50 (LPAG); Pdo. La Plata, Isla Martín García, 12-XII-2009, Colares \& Martínez 54
(LPAG). Pluchea sagittalis (Lam.) Cabrera. Buenos Aires: Pdo. Ensenada, Isla Santiago, 14III-2012, Hernández 116 (LPAG). Solidago chilensis Meyen var. chilensis. Buenos Aires: Pdo. Berisso, camino La Plata-Berisso, 29-III2009, Hernández 17 (LPAG); Pdo. La Plata, JBA "C. Spegazzini”, FCAyF, UNLP, 28-II-2010, Arambarri 500 (LPAG); Pdo. Berisso, camino La Plata-Berisso, III-2012, Hernández 100 (LPAG). Sonchus oleraceus L. Buenos Aires: Pdo. de La Costa, San Clemente del Tuyú, s.f., Cabrera 4918 (LP); Pdo. La Plata, La Plata, 22-III-2012, Arambarri 376 (LPAG). Taraxacum officinale G. Weber ex F. H. Wigg. Buenos Aires: Pdo. Berisso, Isla Paulino, 29-III-2009, Hernández 27 (LPAG); Pdo. La Plata, La Plata, I-2012, Arambarri 378 (LPAG); Pdo. Ensenada, Isla Santiago, 14-III-2012, Hernández 58 (LPAG). Xanthium spinosum L. Buenos Aires: Pdo. La Plata, La Plata, 29-XII-1972, Dimitri s.n. (LPAG 3678); Pdo. Berisso, Palo Blanco, 17-V-2009, Hernández 38 (LPAG). Xanthium strumarium L. Buenos Aires: Pdo. Ensenada, Isla Santiago, 14-III-2012, Hernández \& Oviedo 57 (LPAG). BORAGINACEAE: Borago officinalis L. Buenos Aires: Pdo. La Plata, Isla Martín García, 22-X-1992, Hurrell et al. 1255 (LP); Gonnet, 4-X-1998, Delucchi 1846 (LP); Facultad Agronomía, 5-XI-1922, sin colector (LPAG). Echium plantagineum L. Buenos Aires: Pdo. Ensenada, Punta Lara, 16-X-1979, Volkart s. $n$. (LPAG); Pdo. La Plata, Isla Martín García, 22-X1992, Hurrell et al. 1257 (LP); La Plata, 14-X2011, Arambarri \& Novoa 360 (LPAG); La Plata, JBA“C. Spegazzini”, FCAyF, UNLP, 6-XI-2012, Arambarri \& Vera Bahima 505 (LPAG). BRASSICACEAE: Lepidium bonariense L. Buenos Aires: Pdo. La Plata, La Plata, 11-X2011, Arambarri 366 (LPAG); 14-XI-2011, La Plata, Arambarri 368 (LPAG). Lepidium didymum L. Buenos Aires: Pdo. La Plata, La Plata, 14-XI-2011, Arambarri 364 (LPAG). Raphanus sativus L. Buenos Aires: Pdo. Mar Chiquita, Santa Clara del Mar, 23-I-1973, Dimitri s. n. (LAPG); Pdo. La Plata, La Plata, 5-X-2011, Arambarri 359 (LPAG). CHENOPODIACEAE: Dysphania retusa (Juss. ex Moq.) Mosyakin \& Clemants (=Chenopodium retusum Juss. ex Moq.). Buenos Aires: Pdo. La Plata, Isla Martín García, 13-III-1995, Hurrell et al. 2401 (LP). COMMELINACEAE: Commelina erecta L. 
Buenos Aires: Pdo. La Plata, La Plata, 6-XII2011, Arambarri 370 (LPAG); Pdo. La Plata, JBA “C. Spegazzini”, FCAyF, UNLP, 15-XII2011, Arambarri s.n. (LPAG). Tripogandra diuretica (Mart.) Handlos. Buenos Aires: Pdo. San Fernando, Delta del Río Paraná Miní, 18-V1950, Cabrera 10635 (LP); Pdo. Ensenada, Río Santiago, 18-IV-1964, Cabrera 15914 (LP); Pdo. Berisso, Balneario Bagliardi, 6-V-1995, Bayón 308 (LPAG). CRASSULACEAE: Crassula multicava Lem. Buenos Aires: Pdo. La Plata, La Plata, X-2014, Arambarri 506 (LPAG). EUPHORBIACEAE: Euphorbia serpens Kunth. Buenos Aires: Ciudad Autónoma, 10-II-1966, Amorin s.n. (LPAG); Pdo. La Plata, La Plata, 28XII-2011, Arambarri 375 (LPAG); Pdo. Berisso, Isla Paulino, 20-I-2009, Hernández 11 (LPAG). FABACEAE: Medicago polymorpha L. Buenos Aires: Pdo. La Plata, JBA "C. Spegazzini", FCAyF, UNLP, 27-IX-2011, Arambarri 352 (LPAG). Melilotus indicus (L.) All. Buenos Aires: Pdo. La Plata, La Plata, 11-X-2011, Arambarri 358 (LPAG). Trifolium repens L. Buenos Aires: Pdo. La Plata, JBA "C. Spegazzini”, FCAyF, UNLP, 27-IX-2011, Arambarri 351 (LPAG). FUMARIACEAE: Fumaria capreolata L. Buenos Aires: Pdo. La Plata, La Plata, IX-2004, Monti 14 (LPAG); Pdo. La Plata, JBA “C. Spegazzini”, FCAyF, UNLP, 27-IX-2011, Arambarri 355 (LPAG). GERANIACEAE: Geranium molle L. Buenos Aires: Pdo. La Plata, JBA "C. Spegazzini", FCAyF, UNLP, 11-X-2004, Arambarri 243 (LPAG); 27-IX-2011, Arambarri 354 (LPAG). IRIDACEAE: Sisyrinchium chilense Hook. ssp. chilense. Buenos Aires: Pdo. La Plata, La Plata, JBA “C. Spegazzini”, FCAyF, UNLP, 8-IX-1995, Colares 46 (LPAG); La Plata, 11-X-2011, Arambarri 357 (LPAG). LAMIACEAE: Cantinoa mutabilis (Rich.) Harley \& J.F.B. Pastore. Buenos Aires: Pdo. Ramallo, s.1., 22XII-2001, Torres Robles 623 (LP). Mentha $\boldsymbol{x}$ piperita L. Buenos Aires: Pdo. Berisso, Isla Paulino, 20-I-2009, Hernández 13 (LPAG); Pdo. Ensenada, Isla Santiago, 21-III-2012, Hernández \& Novoa 56 (LPAG); Pdo. La Plata, Estación Experimental “J. Hirschhorn", FCAyF, UNLP, 15-III-2013, Ringuelet s.n. (LPAG 9991). Ocimum basilicum L. Buenos Aires: Pdo. La Plata, La Plata, Estación Experimental “J. Hirschhorn" FCAyF, UNLP, X-2013, Nughes s.n.
(LPAG). Origanum vulgare L. Buenos Aires: Pdo. Berisso, Isla Paulino, 5-XII-2009, Hernández 2 (LPAG); Pdo. La Plata, La Plata, 26-VIII-2013, Nughes s.n. (LPAG); Pdo. Ensenada, Isla Santiago, IX-2012, Hernández 42 (LPAG). Rosmarinus officinalis L. Buenos Aires: Pdo. La Plata, La Plata, 26-VIII-2013, Colares s.n. (LPAG); Pdo. Berisso, Isla Paulino, 17-V-2009, Hernández 37 (LPAG); Pdo. Ensenada, Isla Santiago, 14-III-2012, Hernández 47 (LPAG). Salvia officinalis L. Buenos Aires: Pdo. Berisso, Isla Paulino, 5-XII-2009, Hernández 4 (LPAG). MALVACEAE: Malva parviflora L. Buenos Aires: Pdo. La Plata, Isla Martín García, 25-X-1992, Hurrell et al. 1304 (LP). Modiola caroliniana (L.) G. Don. Buenos Aires: Pdo. La Plata, La Plata, 28-X-2002, Martínez 10 (LPAG). Sida rhombifolia L. Buenos Aires: Pdo. Ensenada, Punta Lara, 5-IV1940, Cabrera 6342 (LP). Pdo. Berisso, Isla Paulino, 29-III-2009, Hernández 18 (LPAG). ONAGRACEAE: Oenothera affinis Cambess. Buenos Aires: Pdo. Campana, Campana, 26-III2003, Delucchi 2694 (LP); Pdo. Magdalena, Paraje El Pino, 13-IV-1995, Bayón 255 (LPAG). OXALIDACEAE: Oxalis articulata Savigny ssp. articulata.Buenos Aires: Pdo. La Plata, La Plata, 27-IX-2011, Arambarri 350 (LPAG). PHYLLANTHACEAE: Phyllanthus niruri L. Buenos Aires: Pdo. La Plata, JBA "C. Spegazzini”, FCAy F, UNLP, IX-2011, Arambarri 409 (LPAG). Entre Ríos: Dpto. Paraná, Paraná, 10-XII-2011, Albornoz s.n. (LPAG). PLANTAGINACEAE: Plantago lanceolata L. Buenos Aires: Pdo. La Plata, Los Porteños, 11III-1995, Bayón 165 (LPAG). P. major L. Buenos Aires: Pdo. La Plata, La Plata, XII-2011, Arambarri 375 (LPAG); Pdo. Ensenada, Isla Santiago, 14-III-2012, Hernández 106 (LPAG). P. tomentosa Lam. ssp. tomentosa. Buenos Aires: Pdo. La Plata, Los Hornos, 19-III-1995, Bayón 212; 219 (LPAG). POACEAE: Cymbopogon citratus (DC.) Stapf. Buenos Aires; Pdo. La Plata, JBA "C. Spegazzini", FCAyF, UNLP, 28-X-2014, Arambarri 504 (LPAG). PORTULACACEAE: Portulaca oleracea L. Buenos Aires: Pdo. La Plata, JBA "C. Spegazzini", FCAyF, UNLP, 28-XII-2011, Arambarri 374 (LPAG); Pdo. Ensenada, Isla Santiago, 18-IV-2012, Hernández 122 (LPAG). SCROPHULARIACEAE: Verbascum virgatum 
Stokes. Buenos Aires: Pdo. La Plata, JBA “C. Spegazzini”, FCAyF, UNLP, XII-2013, Arambarri 502 (LPAG). SOLANACEAE: Physalis viscosa L. Buenos Aires: Pdo. Gral. Madariaga, campo de Aranziaga, 16-II-1947, Rodrigo 3542 (LP); Pdo. La Plata, La Plata, 21-II-1995, Bayón 113 (LPAG). Salpichroa origanifolia (Lam.) Baill. Buenos Aires: Pdo. La Plata, Joaquín Gorina, 18-II-1995, Bayón 103 (LPAG); La Plata, JBA “C. Spegazzini”, FCAyF, UNLP, 27-IX-2011, Arambarri 353(LPAG).Solanum sisymbriifolium Lam. Buenos Aires: Pdo. La Plata, isla Martín García, 11-XI-1992, Hurrell 1348 (LP); La Plata, 17-VIII-2014, Arambarri 507 (LPAG). URTICACEAE: Parietaria debilis G. Forst. Buenos Aires: Pdo. La Plata, La Plata, 11-XI2011, Arambarri 365 (LPAG). Urtica urens L. Buenos Aires: Pdo. La Plata, La Plata, 11-XI2011, Arambarri 362, 363 (LPAG); Pdo. Lobería, Lobería, s.f., Scala 1918 (LP). VERBENACEAE: Phyla nodiflora (L.) Greene var. minor (Gillies \& Hook. ex Hook.) N. O'Leary \& P. Peralta. Buenos Aires: Pdo. La Plata, La Plata, 15-XI2011, Arambarri 369a; 28-XI-2011, Arambarri $369 b$ (LPAG). Verbena bonariensis L. var. bonariensis. Buenos Aires: Pdo. La Plata, La Plata, 28-IV-2010, Arambarri 356 (LPAG). Verbena litoralis Kunth var. litoralis. Buenos Aires: Pdo. Magdalena, Reserva "El Destino", 30-X-2010, Torres Robles et García 983 bis (LP); Pdo. Ensenada, Isla Santiago, 14-II-2012, Hernández 105 (LPAG). Verbena montevidensis Spreng. Buenos Aires: Pdo. Tigre, Don Torcuato, 1-III- 1984, Orfila s. n. (LPAG); Pdo. La Plata, La Plata, Bayón 190 (LPAG); Pdo. San Pedro, Rincón de San Pedro Dávila de los Arrecifes, XI2004, Torres Robles \& Voglino 2402 (LP); Pdo. Berisso, Palo Blanco, 14-IV-1929, Cabrera 861 (LP).

Anatomía, análisis químico y microscopía óptica

Para los estudios histológicos, se emplearon hojas adultas. Las muestras de hoja de los ejemplares de herbario fueron reconstituidas en un recipiente cerrado que contenía agua con una gota de detergente, el cual se colocó en estufa a $30^{\circ} \mathrm{C}$ por $24-$ 72 horas. Luego, al igual que el material fresco se conservó en solución de formalinaaceto-alcohólica (FAA 70 \%) (Johansen,
1940). Para el estudio de las epidermis se practicaron diferentes técnicas: impronta con goma adhesiva, "peeling" y se diafanizaron en frascos de vidrio con tapa de plástico a temperatura ambiente, sumergiendo las hojas en una mezcla en partes iguales de hidróxido de sodio al 5\% e hipoclorito de sodio al 5\%. Una vez decoloradas las láminas se lavaron repetidas veces sumergiéndolas un mínimo de $24 \mathrm{~h}$ en hidrato de cloral. Para analizar la estructura de las láminas, se hicieron cortes transversales a mano alzada y con micrótomo de congelación que abarcaron la parte media de la vena media y alas. Una vez elegidos los cortes, fueron decolorados y se realizaron diversas tinciones facilitando la identificación de las estructuras. Se empleó safranina alcohólica $80 \%$, doble coloración con azul de Alcian y safranina y el colorante metacromático violeta de cresilo al 1\%. En todos los casos se montaron en gelatinaglicerina. Los parámetros micrográficos cuantitativos surgieron del recuento de las células epidérmicas, estomas y tricomas existentes en ambas epidermis en vista frontal y en cuatro campos ubicados en la parte media de cada semilimbo. Las células y estomas que se encontraban en el borde del área de observación y con menos de la mitad dentro del área, no fueron consideradas. Se determinaron densidad de células, estomas y tricomas por unidad de superficie $\left(\mathrm{mm}^{2}\right)$ y se calcularon los índices estomático y de tricomas. Para el cálculo del índice estomático se aplicó la fórmula de Salisbury (1927): [nro. de estomas / (nro. de estomas + nro. células epidérmicas)] x 100. La misma fórmula pero reemplazando los estomas por los pelos o tricomas se empleó para calcular el índice de tricomas o índice piloso, ambos expresados en porcentaje (\%) (Hernández \& Albornoz, 2001). En la vista frontal de la epidermis, se determinaron los caracteres cualitativos (ondulación de las paredes anticlinales de las células epidérmicas, tipos de estomas y de tricomas) y en los cortes transversales de la lámina las caras de la vena media y su estructura, tipo de mesofilo, cristales y estructuras secretoras. Se practicaron técnicas histoquímicas para identificar almidón con solución acuosa 
de Lugol (iodo-iodurada de potasio) (Ruzin, 1999); para sustancias lipofílicas se empleó una solución alcohólica saturada de Sudan IV (Johansen, 1940); las células de mirosina con reactivo de Millon (Zarlavsky, 2014). Para mucílagos se realizó el ensayo fitoquímico (test de precipitado en alcohol) usando alcohol absoluto (Korwar et al., 2010). Las estructuras fueron estudiadas con microscopio óptico (MO) Leitz SM Lux. Las figuras fueron preparadas con MO Gemalux equipado con cámara Motic 1000 y software Motic Image Plus 2.0. La terminología utilizada está de acuerdo con Stace (1965) y Metcalfe \& Chalk (1979). Para la nomenclatura botánica se consideró a Zuloaga et al. (2008) URL: www.darwin. edu.ar; Tropicos.org e Integrated Taxonomic Information System (ITIS), United States Department of Agriculture (USDA), (consultados noviembre 2014). En la Tabla 1 se indican para cada especie los parámetros cuantitativos de la hoja. En el Apéndice 1 se presentan datos tomados de la bibliografía brindando para cada una de las especies estudiadas nombre vulgar, tipo y venación de la hoja, el uso popular y la actividad biológica.

\section{Resultados}

En la vista frontal de la epidermis más del $80 \%$ de las especies presentaron las paredes anticlinales de las células onduladosinuosas a sinuosas, la cutícula delgada (0,5-1,5 $\mu \mathrm{m}$ de espesor). Los estomas se ubicaron a nivel o elevados con respecto a las restantes células epidérmicas. El 79\% de las especies tuvieron láminas foliares anfistomáticas, mientras que en Achyrocline satureioides, Fumaria capreolata, Galinsoga parviflora, Mikania micrantha, Oxalis articulata, Parietaria debilis, Phyllanthus niruri, Rosmarinus officinalis, Sisyrinchium chilense var. chilense, Tripogandra diuretica, Urtica urens, Verbena bonariensis y Vinca major fueron hipostomáticas. En el $80 \%$ de las especies los estomas fueron anomocíticos o anomocíticos y anisocíticos. En Alternanthera spp., Plantago lanceolata y $P$. major se hallaron los tipos anomocíticos y diacíticos; siendo estos últimos característicos de la familia Lamiaceae. Los estomas paracíticos caracterizaron las especies Cymbopogon citratus, Phyllanthus niruri y Vinca major, ocasionalmente se encontraron en Euphorbia serpens; se hallaron estomas ciclocíticos en Crassula multicava, paralelocíticos en Portulaca oleracea, hexacíticos en Commelina erecta; tetracíticos en Tripogandra diuretica y ocasionalmente en Dysphania retusa. El 45\% de las especies, en la cara abaxial y el $35 \%$ en la cara adaxial tuvieron un índice estomático entre el 10 y $20 \%$. El segundo valor de IE más numeroso, presente en el 31 al $34 \%$ de las especies estudiadas, osciló en la cara abaxial entre el 20 y $30 \%$ y en la cara adaxial resultó inferior al 10\% (Tabla 1). Las hojas del $90 \%$ de las especies fueron pubescentes con pelos glandulares y/o eglandulares. Las epidermis glabras se hallaron en Acmella decumbens, Crassula multicava, Euphorbia serpens, Fumaria capreolata, Phyllanthus niruri y Sonchus oleraceous. Entre el 52 y $58 \%$ de las especies tuvieron un índice piloso inferior al $5 \%$, tanto en la cara adaxial como abaxial (Tabla 1). El mesofilo en el $82 \%$ de las especies fue dorsiventral, pero se encontró isolateral en Conyza bonariensis, Dysphania retusa, y Solidago chilensis var. chilensis; homogéneo en Crassula multicava, Cymbopogon citratus, Portulaca oleracea y Sisyrinchium chilense var. chilense y con alta variabilidad, dorsiventral, homogéneo o isolateral en Cichorium intybus, Plantago lanceolata, P. major, Sonchus oleraceus y Taraxacum officinale. La vena media en corte transversal es en su cara adaxial plana, cóncava o convexa y en su cara abaxial plano o convexa, es decir, vena biplana, cóncavo-convexa o biconvexa. Este carácter de la vena media junto a otros como tipo de estomas, tricomas, cristales, reservorios, sustancias químicas y los índices estomático y piloso permitieron la elaboración de la clave dicotómica. 


\section{Clave para diferenciar 62 especies de hierbas terrestres con propiedades medicinales de la Región Rioplatense (Buenos Aires, Argentina)}

1. Epidermis en vista frontal con el eje longitudinal de los estomas ubicado paralelo al eje longitudinal de la lámina.

2. Estomas paracíticos, las células oclusivas con forma de pesas. Haces vasculares con estructura kranz. Presencia de gotas de sustancias lipofílicas en el mesofilo.

1. Cymbopogon citratus (Fig. 1A, B)

2'. Estomas anomocíticos, tetra o hexacíticos, las células oclusivas con forma de riñón. Haces vasculares sin estructura kranz. Ausencia de gotas de sustancias lipofílicas en el mesofilo.

3. Estomas anomocíticos, hundidos. Células epidérmicas con paredes engrosadas. Ausencia de cristales.

2. Sisyrinchium chilense var. chilense (Fig. 1C)

3'. Estomas tetracíticos o hexacíticos. Células epidérmicas con paredes delgadas. Presencia de cristales.

4. Presencia de pelos eglandulares uncinulados.

3. Commelina erecta (Fig. 1D)

4 '. Ausencia de pelos eglandulares uncinulados.

4. Tripogandra diuretica

1'. Epidermis en vista frontal con el eje longitudinal de los estomas ubicado al azar respecto al eje longitudinal de la lámina.

5. Células epidérmicas de la cara adaxial en vista frontal con paredes anticlinales rectilíneas. Cutícula gruesa $(2,2-2,7 \mu \mathrm{m}$ de espesor).

5. Rosmarinus officinalis

5 '.Células epidérmicas en vista frontal con paredes anticlinales curvadas, ondulado-sinuosas o sinuosas. Cutícula delgada $(0,5-1,5 \mu \mathrm{m}$ de espesor).

6. Parénquima del mesofilo con rafidios, nunca drusas.

6. Oenothera affinis

6'. Parénquima del mesofilo con drusas, nunca rafidios.

7. Presencia de haces vasculares con estructura kranz.

8. Epidermis glabra. Vena media en corte transversal, biplana o plano-convexa.

9. Vena media biplana. Estomas paralelocíticos. Presencia de drusas y cavidades mucilaginíferas. Ausencia de tubos laticíferos.

7. Portulaca oleracea

9'. Vena media plano-convexa. Estomas paracíticos, anomocíticos y anisocíticos. Ausencia de drusas y cavidades mucilaginíferas. Presencia de tubos laticíferos.

8. Euphorbia serpens (Fig. 1E, F)

8'. Epidermis pubescente. Vena media en corte transversal biconvexa o cóncavo-convexa.

10. Vena media biconvexa. Presencia de drusas.

9. Alternanthera philoxeroides f. philoxeroides

10. Alternanthera pungens

10'. Vena media cóncavo-convexa. Presencia de drusas y arena cristalina.

11. Amaranthus hybridus ssp. hybridus

7'. Ausencia de haces vasculares con estructura kranz. 
11. Vena media en corte transversal biconvexa.

12. Presencia de drusas.

13. Epidermis glabra, papilosa. Estomas paracíticos. Ausencia de cavidades mucilaginíferas.

12. Phyllanthus niruri (Fig. 2A)

13'. Epidermis pubescente, nunca papilosa. Estomas anomocíticos y anisocíticos. Presencia de cavidades mucilaginíferas.

14. Vena media en corte transversal biconvexa, sin formar prominencia adaxial. Tricomas estrellados: densidad 331,36 tricomas $/ \mathrm{mm}^{2}$. Índice piloso abaxial 9,26\%.

13. Sida rhombifolia (Fig. 2B)

14'. Vena media en corte transversal biconvexa, muy prominente en la cara adaxial. Tricomas estrellados: densidad inferior a 100 tricomas $/ \mathrm{mm}^{2}$. Índice piloso abaxial entre 2 y $4 \%$.

15. Mesofilo dorsiventral con una capa de parénquima en empalizada. Tricomas estrellados: densidad 53,03 tricomas $/ \mathrm{mm}^{2}$. Índice piloso abaxial 3,06\%.

14. Malva parviflora

15'. Mesofilo dorsiventral con 3-4 capas de parénquima en empalizada. Tricomas estrellados: densidad 30,30 tricomas $/ \mathrm{mm}^{2}$. Índice piloso abaxial 2,35\%.

15. Modiola carolineana

$12^{\prime}$. Ausencia de drusas.

16. Estomas paracíticos o diacíticos.

17. Estomas paracíticos. Lámina hipostomática. Presencia de haces bicolaterales. Presencia de tubos laticíferos.

16. Vinca major (Fig. 2C, D)

17'. Estomas diacíticos. Lámina anfistomática. Presencia de haces colaterales. Ausencia de tubos laticíferos.

18. Tricomas eglandulares en cojín. Índice estomático adaxial no mayor al $6 \%$.

17. Cantinoa mutabilis (Fig. 2E)

18'. Tricomas eglandulares no en cojín. Índice estomático adaxial y abaxial superior a $20 \%$.

18. Ocimum basilicum

16'. Estomas anomocíticos y anisocíticos.

19. Presencia de arena cristalina.

20. Haces vasculares colaterales. Presencia de tricomas glandulares vesiculosos.

19. Dysphania retusa (Fig. 2F)

20 '. Haces vasculares bicolaterales. Ausencia de tricomas glandulares vesiculosos.

21. Tricomas estrellados con varias ramas.

20. Solanum sisymbriifolium

$21^{\prime}$. Tricomas no estrellados. Tricomas simples o ramificados.

22. Tricomas ramificados.

21. Physalis viscosa

22'. Tricomas no ramificados.

22. Salpichroa origanifolia

19 '. Ausencia de arena cristalina.

23. Presencia de reservorio esquizógeno del lado abaxial, junto al tejido vascular.

24. Epidermis glabra.

23. Acmella decumbens 
24'. Epidermis pubescente.

25. Mesofilo isolateral.

26. Mesofilo con 1-2 capas en empalizada hacia la epidermis adaxial y solo una capa abaxial. IE: $11-13 \%$ adaxial y abaxial.

24. Conyza bonariensis

26'. Mesofilo con 2-3 capas en empalizada hacia la epidermis adaxial y otras 2-3 capas abaxiales. IE: $4-5 \%$ adaxial y abaxial.

25. Solidago chilensis var. chilensis (Fig. 3A)

25'. Mesofilo dorsiventral.

27. Mesofilo con una capa en empalizada adaxial.

28. Lámina hipostomática. IE abaxial: 7\%. IP abaxial: 0,28\%.

26. Galinsoga parviflora (Fig. 3B)

28'. Lámina anfistomática. IE abaxial: 21\%. IP abaxial: 2,71\%.

27. Bidens pilosa

27'. Mesofilo con dos capas en empalizada adaxial.

29. Lámina hipostomática. Epidermis abaxial con IP: 3,58 \% .

28. Mikania micrantha

29'. Lámina anfistomática. Epidermis abaxial con IP abaxial de 0,49 \% hasta más de $10 \%$.

30. Epidermis abaxial con IP: $0,49 \%$.

29. Xanthium strumarium

30'. Epidermis abaxial con IP: mayor a 10\%.

30. Xanthium spinosum

23'. Ausencia de reservorio esquizógeno del lado abaxial junto al tejido vascular.

31. Presencia de tubos laticíferos.

31. Taraxacum officinale

31'. Ausencia de tubos laticíferos.

32. Estomas anomocíticos. Tricomas eglandulares y glandulares presentes. Mesofilo isolateral con una capa en empalizada hacia cada epidermis.

32. Pluchea sagittalis (Fig. 3C, D)

32'. Estomas anisocíticos. Tricomas eglandulares unicelulares, agudos, escasos y glandulares ausentes. Mesofilo dorsiventral con tres capas en empalizada adaxial.

33. Raphanus sativus (Fig. 3E)

11'. Vena media en corte transversal cóncavo-convexa, plano-convexa o biplana.

33. Vena media biplana. Estomas ciclocíticos.

34. Crassula multicava (Fig. 3F)

33'. Vena media cóncavo-convexa o plano-convexa. Estomas de otro tipo.

34. Epidermis glabra.

35. Lámina hipostomática con IE abaxial de 21,14\%. Estomas anomocíticos. Presencia de cristales. Vena media con clorénquima adaxial.

35. Fumaria capreolata

35'. Lámina anfistomática con IE abaxial de 18,00\%. Estomas anomocíticos y anisocíticos. Ausencia de cristales. Vena media con aerénquima adaxial.

36. Sonchus oleraceous (Fig. 4A) 
34'. Epidermis pubescente.

36. Tricomas glandulares, pluricelulares y pluriseriados. Presencia de tubos laticíferos.

37. Cichorium intybus (Fig. 4B, b)

36'. Tricomas con otras características. Ausencia de tubos laticíferos.

37. Estomas diacíticos o anomocíticos y diacíticos.

38. Tricomas glandulares con cabeza bicelular. Vena media con endodermis conspicua.

39. Índice Estomático adaxial y abaxial $23-24 \%$.

38. Plantago tomentosa ssp. tomentosa (Fig. 4C) 39'. Índice estomático adaxial $17-18 \%$ y abaxial $21 \%$.

39. Plantago major

38'. Tricomas glandulares con cabeza pluricelular.

40. Tricomas eglandulares con unión celular ensamblada o ahorquillada.

40. Plantago lanceolata

$40^{\prime}$. Tricomas eglandulares nunca con unión celular ensamblada o ahorquillada.

41. Índice estomático en la cara adaxial 8,81\% y en la abaxial $17-18 \%$. Índice piloso adaxial y abaxial $10-18 \%$.

41. Salvia officinalis

41 '. Índice estomático en la cara adaxial menor al 1\% y en la abaxial 19-20\%. Índice piloso adaxial y abaxial menor al $10 \%$.

42. Índice piloso adaxial y abaxial menor al $2 \%$.

42'. Índice piloso adaxial y abaxial 4 - 7\%.

42. Mentha x piperita

43. Origanum vulgare

37'. Estomas anomocíticos y anisocíticos.

43. Presencia de cistolitos en la epidermis adaxial.

44. Presencia de tricomas uncinulados.

44. Parietaria debilis (Fig. 5A)

44'. Presencia de otro tipo de tricomas, no uncinulados.

45. Presencia de tricomas urticantes.

45. Urtica urens

45'. Ausencia de tricomas urticantes. Tricomas eglandulares con o sin células basales con cistolitos.

46. Borago officinalis (Fig. 5B)

47. Echium plantagineum (Fig. 5C)

43'. Ausencia de cistolitos.

46. Tricomas en $\mathrm{T}$.

48. Phyla nodiflora var. minor (Fig. 5D)

46'. Tricomas de otro tipo, nunca en T.

47. Presencia de reservorios esquizógenos en la proximidad de la vena media, ubicados en posición adaxial y abaxial respecto al haz vascular.

49. Ambrosia tenuifolia

47'. Ausencia de reservorios esquizógenos en la proximidad de la vena media. 
48. Pubescencia abaxial densa, flageliforme.

50. Achyrocline satureioides (Fig. 5E)

48'. Pubescencia nunca flageliforme.

49. Tricomas eglandulares tipo látigo y glandulares biseriados.

51. Gamochaeta coarctata

49'. Tricomas eglandulares y glandulares de otro tipo.

50. Haces vasculares rodeados de vaina cristalífera con maclas.

52. Medicago polymorpha

53. Melilotus indicus

54. Trifolium repens (Fig. 6A)

$50^{\prime}$. Haces vasculares rodeados por vaina parenquimática o endodermis, nunca cristalífera.

51. Presencia de células secretoras.

52. Tricomas eglandulares estrellados estipitados y tricomas glandulares.

55. Verbascum virgatum (Fig. 6B-D)

52'. Tricomas eglandulares nunca estrellados, ni estipitados. Ausencia de tricomas glandulares.

53. Mesofilo dorsiventral. Presencia de mucrón marginal.

56. Lepidium didymum (Fig. 6E)

53'. Mesofilo homogéneo. Ausencia de mucrón marginal.

57. Lepidium bonariense

51'. Ausencia de células secretoras.

54. Mesofilo dorsiventral con una capa de empalizada adaxial.

55. Epidermis papilosa. Mesofilo con cristales agrupados y cavidades secretoras.

58. Oxalis articulata ssp. articulata

55'. Epidermis no papilosa. Mesofilo sin cristales agrupados y sin cavidades secretoras.

59. Geranium molle

54'. Mesofilo dorsiventral con 2-4 capas de empalizada adaxial.

56. Lámina hipostomática. IE abaxial 15-16\%.

60. Verbena bonariensis var. bonariensis

56'. Lámina anfistomática. IE abaxial 18-22\%.

61. Verbena litoralis var. litoralis

62. V. montevidensis

\section{Discusión}

En general los caracteres epidérmicos y estructurales de la hoja resultaron coincidentes con trabajos previos. Sin embargo, en los párrafos siguientes se incluye una discusión más detallada y se anotan algunas características que se citan por primera vez. En la familia Amaranthaceae se estudiaron tres especies, que presentaron mesofilo dorsiventral y estructura kranz como lo indicaron (Metcalfe \& Chalk, 1979; Barboza et al., 2001; Duarte \& Debur, 2004). De acuerdo con los diferentes autores y 10 hallado, todas las especies poseen estomas anomocíticos, pero aparecen acompañados de otros tipos [e.g., en las especies de Alternanthera se hallaron también diacíticos, mientras que no se observaron los paracíticos citados para $A$. pungens por Barboza et al. (2001)] y en Amaranthus hybridus ssp. hybridus se hallaron anisocíticos no 


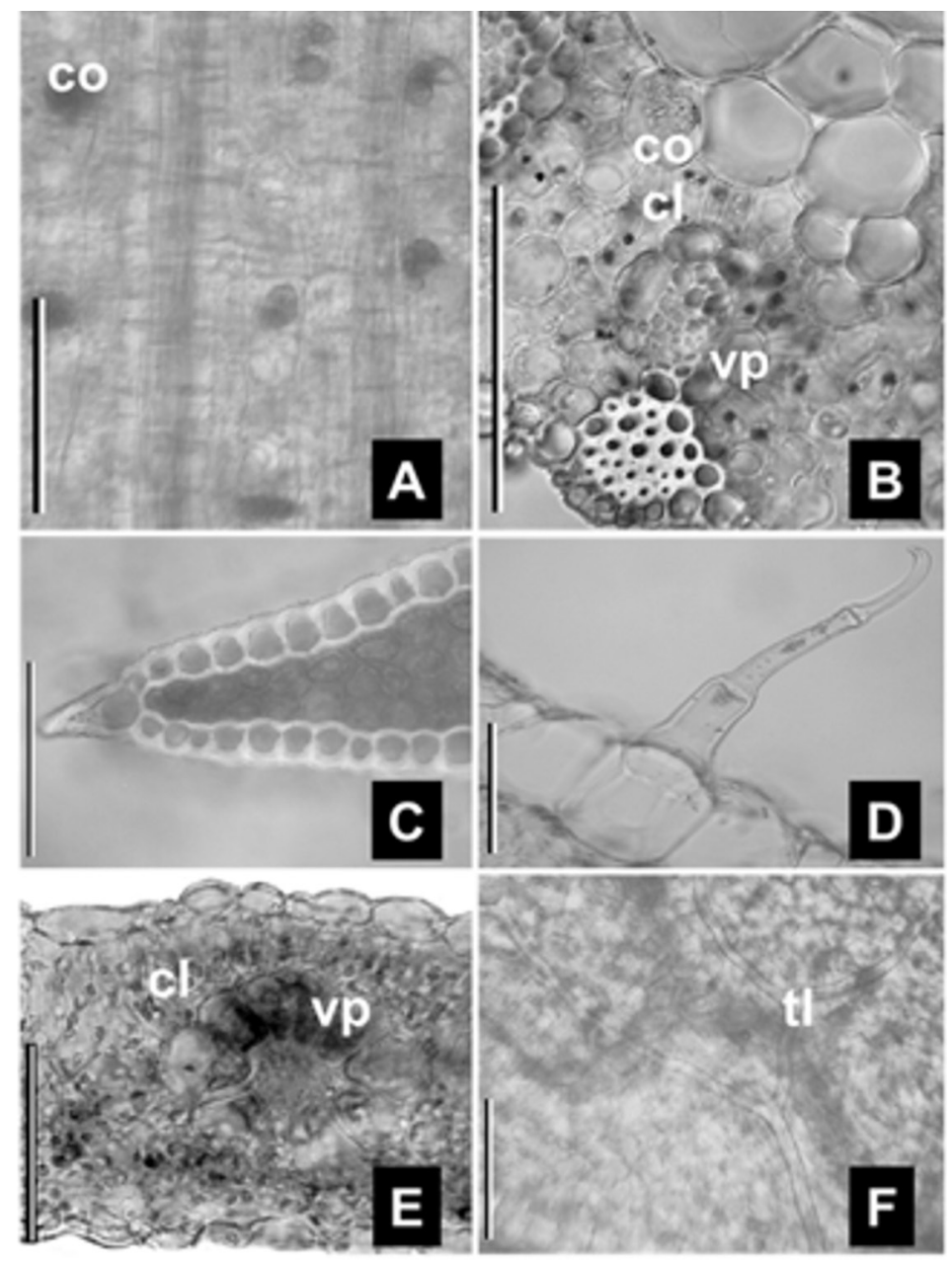

Fig. 1. Cymbopogon citratus. A: Células oleíferas (co) en el mesofilo en vista frontal. B: Corte transversal de hoja mostrando la vaina parénquimática (vp); el clorénquima radiado (cl) de la estructura kranz y una célula oleosa (co). Sisyrinchium chilense var. chilense. C: Células epidérmicas con paredes gruesas y tricoma marginal. Commelina erecta. D: Células epidérmicas con paredes delgadas y un tricoma pluricelular, uniseriado, uncinulado. Euphorbia serpens. E: estructura kranz, vaina parenquimática (vp) con grandes cloroplastos y clorénquima radiado (cl). F: tubos laticíferos no articulados, ramificados (tl). Escalas: $100 \mu \mathrm{m}$.

mencionados con anterioridad. En la familia Apocynaceae se estudió Vinca major cuya epidermis presenta estomas paracíticos, tricomas escasos en el envés, caracteres que acuerdan con Freire et al. (2005); también se observaron el mesofilo dorsiventral, vena media biconvexa con tubos laticíferos en el parénquima del floema como lo señalan Metcalfe \& Chalk (1979). De la amplia familia Asteraceae se analizaron 15 especies, cuyos caracteres coinciden con los estudios previos de raíz y tallo (Arambarri et al., 2013;
Colares et al., 2014), excepto que en la hoja de Pluchea sagittifolia no hay reservorios. Este dato coincide con lo hallado por Cambi et al. (2006) al estudiar detalladamente esta especie. Los caracteres de la hoja de Achyrocline saturioides están de acuerdo con la descripción e ilustración dada por de Gattuso \& Gattuso (1998). Los reservorios y estructura foliar de Mikania micrantha resultaron coincidentes con lo propuesto por Colares (2011) y Amorin et al. (2014) quienes describieron aspectos botánicos y medicinales 


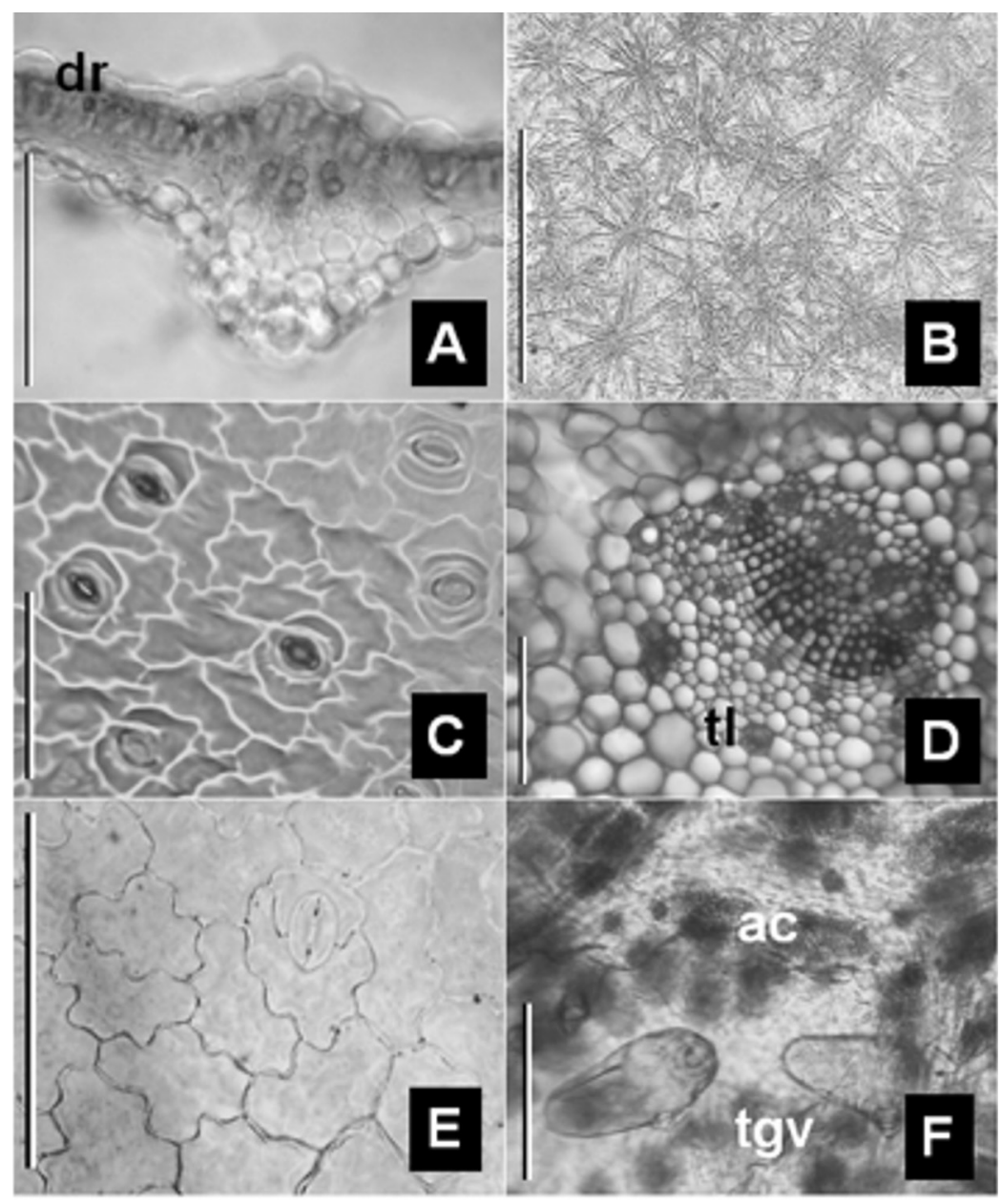

Fig. 2. Phyllanthus niruri. A: Vena media biconvexa, epidermis adaxial y abaxial papilosas, mesofilo dorsiventral, con drusas (dr). Sida rhombifolia. B: Tricomas estrellados en la cara abaxial. Vinca major. C: Estomas paracíticos en la epidermis abaxial. D: haz vascular bicolateral abierto con tubos laticíferos (tl) en el parénquima del floema abaxial. Cantinoa mutabilis. E: Células epidérmicas con paredes anticlinales sinuosas y estoma diacítico. Dysphania retusa. F: Tricomas glandulares vesiculosos (tgv) en la epidermis y arena cristalina (ac) en el mesofilo. Escalas: A, C-F: $100 \mu$ m; B: $300 \mu \mathrm{m}$.

de las especies de Mikania. La estructura de la hoja de Solidago chilensis var. chilensis, fue analizada en un trabajo previo (Hernández et al., 2013). Los caracteres anatómicos de la hoja de Conyza están de acuerdo con la descripción realizada por Perez et al. (2013), asi como ocurrió con los datos sobre Xanthium spinosum brindados por Barboza et al. (2001). En las Brassicaceae las células epidérmicas, tipo de estomas y tricomas de Lepidium bonariense fueron propios de la familia y coincidentes con los analizados por Diorio
(1986). Sin embargo, no hallamos un mesofilo dorsiventral sino homogéneo. También observamos en el parénquima de la vena media, células de mirosina. En L. dydimum (= Coronopus dydimus) encontramos características acordes con las indicadas por Luján \& Barboza (1999) y Barboza et al. (2001). De las Chenopodiaceae se estudió la especie aromática Dysphania retusa, en la cual el tipo de estomas y diversidad de tricomas glandulares coinciden con los datos aportados por Da Silva Filho et al. (1992) y 


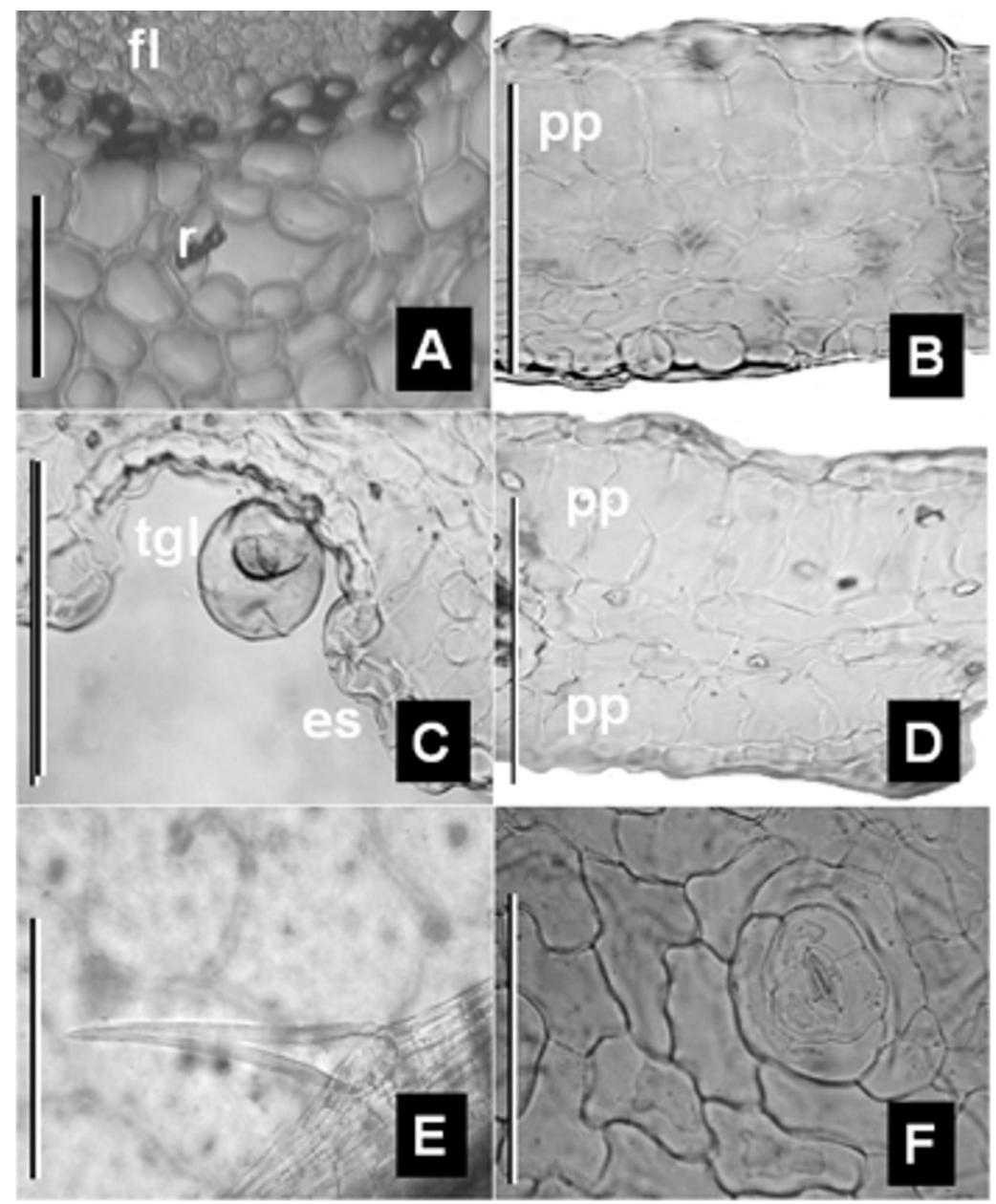

Fig. 3. Solidago chilensis var. chilensis. A: Parte del floema (fl) del haz vascular medio y en su proximidad un reservorio esquizógeno (r). Galinsoga parviflora. B: Corte transversal de la lámina, mesofilo dorsiventral con una capa en empalizada adaxial (pp) y tres estratos de parénquima esponjoso abaxial. Pluchea sagittalis. C: epidermis abaxial de la lámina en corte transveral, con una tricoma glandular (tgl) y estoma elevado (es). D: corte transversal de la lámina, mesofilo isolateral con una capa de parénquima en empalizada (pp) hacia cada epidermis, adaxial y abaxial. Raphanus sativus. E: Tricoma eglandular unicelular. Crassula multicava. F: Epidermis con estoma ciclocítico. Escalas: A: $50 \mu \mathrm{m}$; B-D y F: $100 \mu \mathrm{m}$; E: $300 \mu \mathrm{m}$.

Bonzani et al. (2003). Tanto Da Silva Filho et al. (1992) como Barboza et al. (2001) destacan la similitud de los caracteres de D. retusa con los de Dysphania ambrosioides (= Chenopodium ambrosioides). Con respecto a la secreción de los tricomas glandulares Simon (1987) señaló el aceite esencial ascaridol que le confiere la propiedad aromática y terapéutica y también el efecto tóxico cuando es consumido en dosis excesiva. Los caracteres que hallamos como destacables en esta especie, son los tricomas y la presencia de arena cristalina que contribuyen a su identificación. De las Commelinaceae se estudiaron dos especies, se encontraron estomas de tipo tetracíticos o tetraperígenos en Tripogandra y hexacíticos o hexaperígenos en Commelina, característicos de la familia de acuerdo con Metcalfe \& Chalk (1950) y Fryns-Claessens \& Van Cotthem (1973). La estructura de la lámina de Commelina, coincide con lo descripto e ilustrado por Elb et al. (2010). En Euphorbia serpens (Euphorbiaceae), se hallaron estomas 


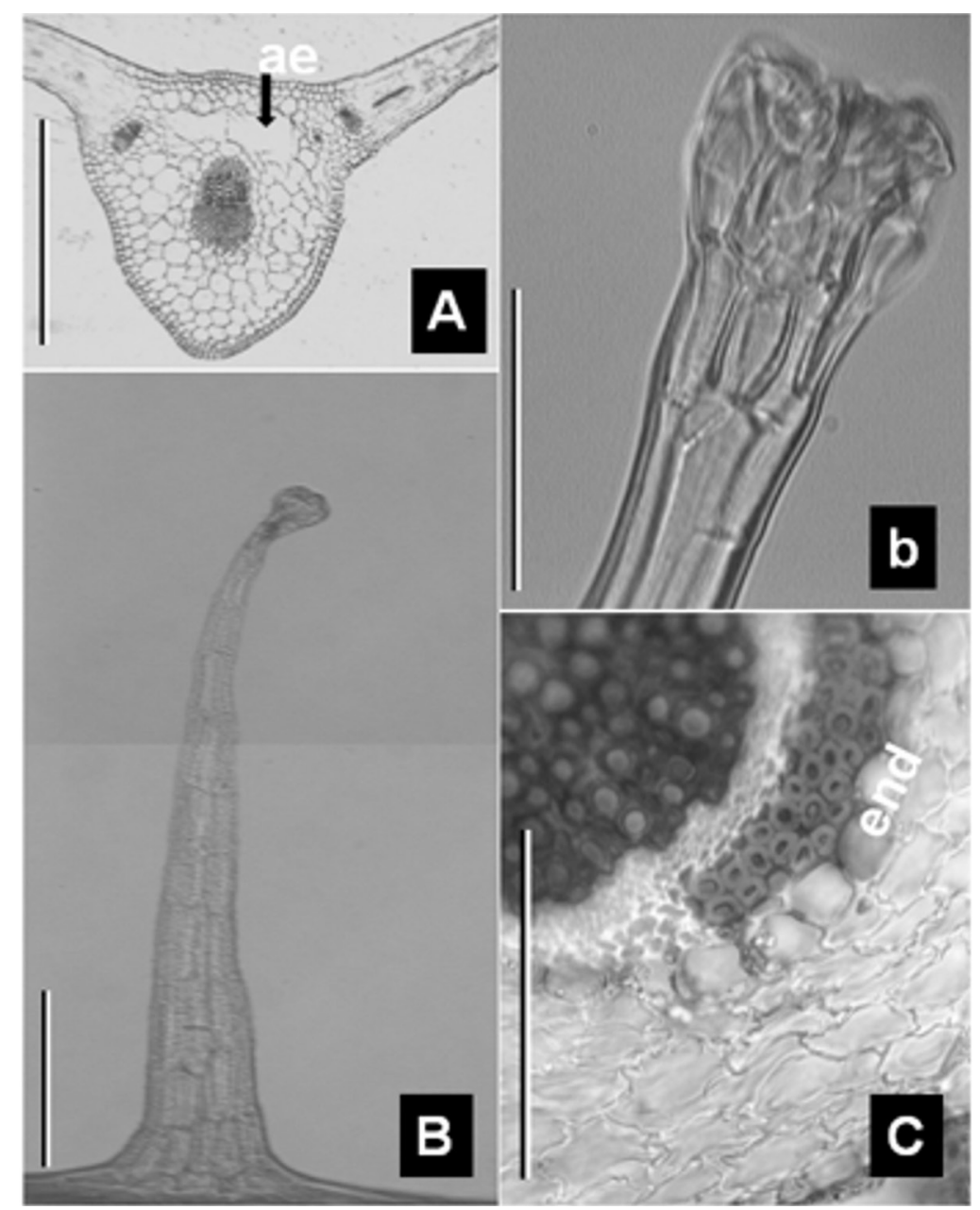

Fig. 4. Sonchus oleraceus. A: Vena media en corte transversal, cóncavo-convexa, con aerénquima (ae) sobre el haz vascular medio. Cichorium intybus. B: tricoma glandular pluricelular, pluriseriado; b, detalle de la cabeza pluricelular. Plantago tomentosa ssp. tomentosa. C: Haz vascular de la vena media, rodeado por endodermis (end) con banda de Caspary. Escalas: A: $1000 \mu \mathrm{m}$; B: $200 \mu \mathrm{m}$; b: $50 \mu \mathrm{m}$; C: $100 \mu \mathrm{m}$.

anomocíticos, anisocíticos y paracíticos, los diferentes tipos de estomas fueron mencionados por Metcalfe \& Chalk (1979). Freire et al. (2005) mencionan que no hallaron estomas anisocíticos y se atribuye a que no todas las Euphorbiaceae los poseen. Las características macro y micromorfológicas foliares de Euphorbia serpens coinciden con lo señalado por Luján \& Barboza (1999) como la estructura kranz e igualmente con lo citado por Aldhebiani \& Jury (2013). De la familia Fabaceae se tratraron tres especies, donde los datos obtenidos son característicos de la misma y coinciden con los descriptos e ilustrados por Yagueddú \& Cid (1992) y Yagueddú et al. (2009). Fumaria capreolata (Fumariaceae) fue analizada de manera comparativa con su similar $F$. officinalis por Luján et al. (2004). En general, los caracteres anatómicos hallados están de acuerdo con los aportados por dichos autores. Sin embargo, encontramos a Fumaria capreolata hipostomática, no anfistomática, lo cual puede estar en relación con una diferente exposición a la radiación solar (Nughes et al., 2013). En Sisyrinchium chilense var. chilense (Iridaceae) las hojas totalmente conduplicadas presentan mesofilo invertido, parénquima 


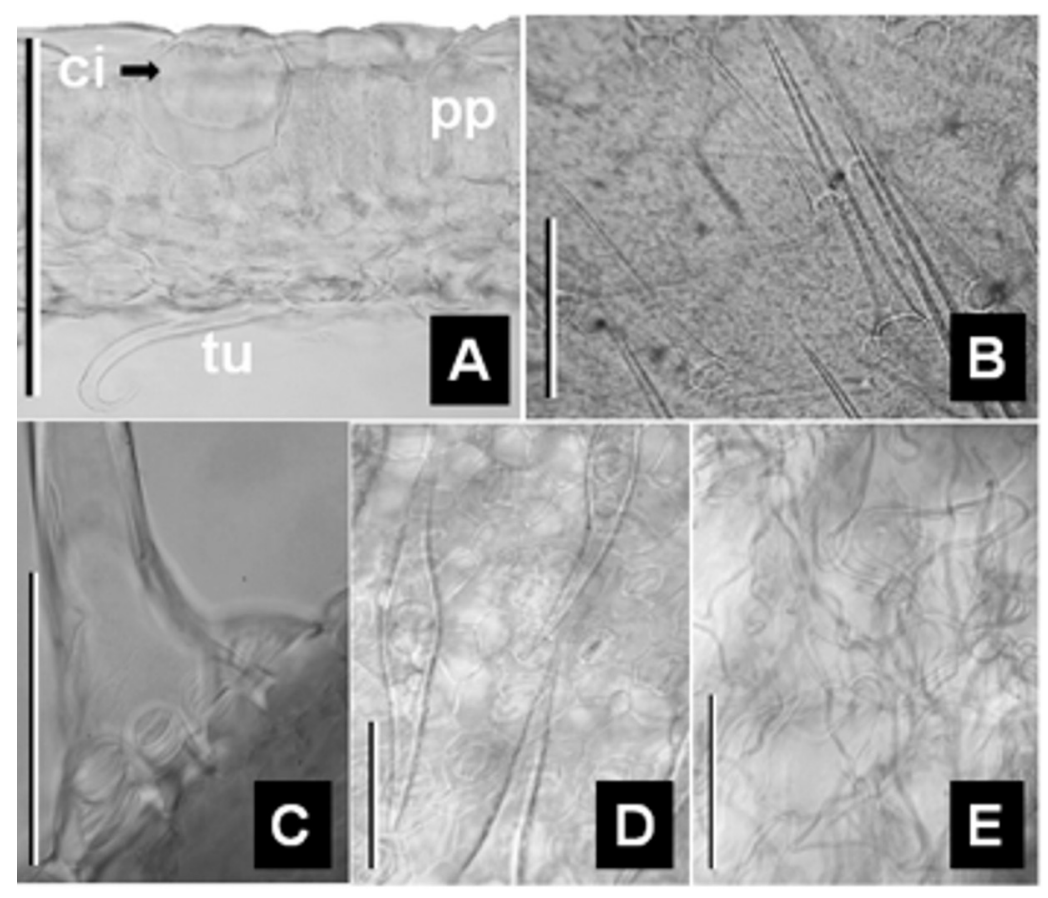

Fig. 5. Parietaria debilis. A: Lámina en corte transversal mostrando cistolito (ci) adaxial, mesofilo dorsiventral con una capa en empalizada (pp) y tricomas uncinulados (tu) en la epidermis abaxial. Borago officinalis. B: Tricomas eglandulares, unicelulares, agudos. Echium plantagineum. C: Base con cistolitos de un tricoma eglandular. Phyla nodiflora var. minor. D: Tricomas en T, rugosos. Achyrocline satureioides. E: Pubescencia flageliforme densa sobre toda la superficie. Escalas: A, C-E: $100 \mu \mathrm{m}$; B: $300 \mu \mathrm{m}$.

incoloro adaxial y parénquima clorofiliano abaxial; la epidermis adaxial o interna muestra paredes delgadas, mientras que la epidermis de la cara externa (abaxial), muestra características xeromórficas, sus células son alargadas en sentido perpendicular a la superficie, poseen paredes notablemente engrosadas y los estomas están hundidos. Algunos de estos rasgos fueron señalados en Vizcaíno et al. (1998). Entre las especies de la familia Lamiaceae, Rosmarinus officinalis presentó caracteres xeromórficos, tales como hojas lineares, con margen revoluto y estomas abaxiales en criptas, características similares a la hoja tipo ericoide de Ancibor (1992). Esta fue la única especie cuya epidermis en corte transversal de la lámina mostró cutícula gruesa mayor a $2 \mu \mathrm{m}$ de espesor y el mesofilo hacia la cara adaxial está formado por varias capas de colénquima seguido hacia la cara abaxial de clorénquima en empalizada. De la familia Malvaceae se estudiaron tres especies, cuya anatomía muestra una gran uniformidad con la presencia de pelos estrellados en la epidermis y cavidades mucilaginíferas en el parénquima foliar (Metcalfe \& Chalk, 1950). En Malva parviflora la descripción completa de la hoja se corresponde con la realizada por Giberti (1981) a la que agregamos la presencia de estomas anisocíticos acompañando los anomocíticos. Oenothera affinis (Onagraceae), posee como carácter destacable la presencia de rafidios ya señalada para la familia por Metcalfe \& Chalk (1950). La especie Oxalis articulata (Oxalidaceae) analizada se corresponde en los caracteres epidérmicos con lo señalado por Freire et al. (2005) y Hadid et al. (2011). Sin embargo, en esta oportunidad se hallaron también estomas anisocíticos. Phyllanthus niruri (Phyllathaceae) presenta una lámina glabra, hipostomática, con estomas paracíticos $\mathrm{y}$ mesofilo dorsiventral conteniendo numerosas drusas como lo señalaron Degen et al. (2011) en su estudio de tres especies medicinales comercializadas en Paraguay. En la familia 


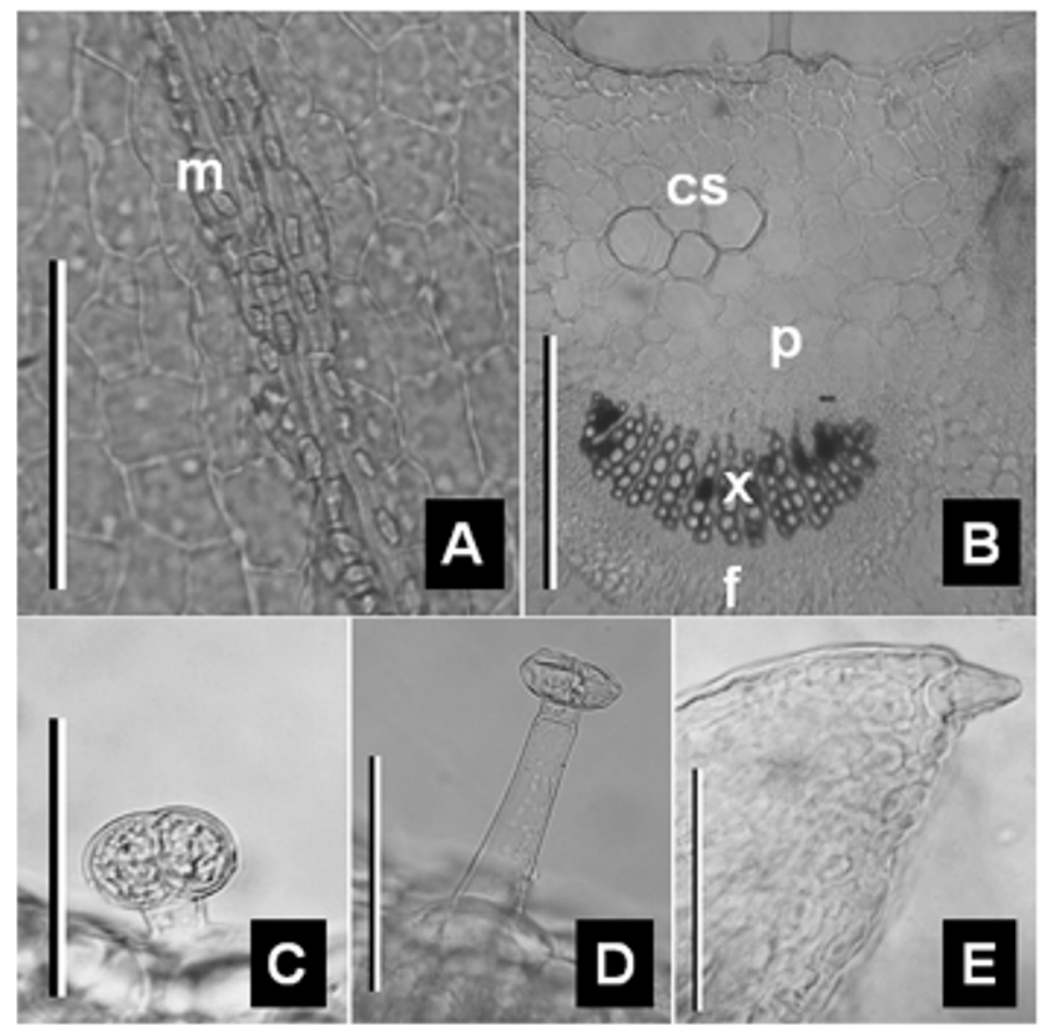

Fig. 6. Trifolium repens. A: Maclas (m) en la vaina parenquimática de las venas. Verbascum virgatum. B: Vena media en corte transversal mostrando células secretoras (cs) en el parénquima (p) sobre el haz vascular (x, xilema; f, floema). C: Ricoma glandular de pie corto, cabeza pluricelular. D: Tricoma glandular de pie largo, cabeza pluricelular. Lepidium didymum. E: Corte transversal de la hoja mostrando el mucrón marginal. Escalas: A, D, E: $100 \mu \mathrm{m} ; \mathrm{B}: 300 \mu \mathrm{m} ; \mathrm{C}: 50 \mu \mathrm{m}$.

Plantaginaceae, las tres especies de Plantago estudiadas coincidieron con las descripciones realizadas por Filippa et al. (1999) y Barboza et al. (2001). Sin embargo, en Plantago lanceolata se encontró un mesofilo homogéneo como lo señalaron Bayón et al. (2000), mientras que Filippa et al. (1999) indican para la misma especie un mesofilo isolateral. Estas diferencias estarían dadas por la plasticidad fenotípica del género y gran variabilidad que presenta la estructura del mesofilo en relación con el ambiente (Metcalfe \& Chalk, 1950). De la familia Poaceae en la especie aromática Cymbopogon citratus se hallaron microtricos y pelos bicelulares con la célula terminal oblongo alargada con pared ténue; sus haces vasculares poseen estructura kranz y en el mesofilo abundan las células oleíferas. De las Portulacaceae se estudio la lámina crasa de
Portulaca oleracea, la epidermis es uniseriada, en vista frontal presenta paredes sinuosas y estomas paralelocíticos, con escasos tricomas papilosos y en su interior un mesofilo homogéneo con estructura kranz como lo indicaron Figueroa et al. (2011, 2013). La vena media en esta especie es biplana y en su mesofilo presenta drusas y cavidades mucilaginíferas. Entre las Scrophulariaceae se analizó la lámina foliar de Verbascum virgatum, anfistomática, los estomas hallados fueron anomo- y anisocíticos, no se observaron paracíticos y hemiparacíticos como señalan para $V$. thapsus L. (Barboza et al., 2001), mientras que los caracteres de vena media y mesofilo resultaron coincidentes en ambas especies. De las tres especies de Solanaceae estudiadas, los resultados están de acuerdo con Colares et al. (1999) y Stenglein (2001). 
Tabla 1. Características epidérmicas vistas en superficie. Densidades $(\mathrm{d} / \mathrm{mm} 2)$ de células epidérmicas (CEL), estomas (EST), tricomas (TRI). Índice Estomático (IE); Índice Piloso (IP) en porcentaje (\%).

\begin{tabular}{|c|c|c|c|c|c|c|c|c|c|c|}
\hline \multirow[b]{2}{*}{ TAXA / CARACTERES } & \multicolumn{3}{|c|}{ EPIDERMIS } & \multicolumn{2}{|c|}{ ADAXIAL } & \multicolumn{3}{|c|}{ EPIDERMIS } & \multicolumn{2}{|c|}{ ABAXIAL } \\
\hline & $\begin{array}{c}\text { CEL } \\
\text { d/mm2 }\end{array}$ & $\begin{array}{c}\text { EST } \\
\mathrm{d} / \mathrm{mm} 2\end{array}$ & $\begin{array}{c}\text { TRI } \\
\text { d/mm2 }\end{array}$ & $\begin{array}{l}\text { IE } \\
\%\end{array}$ & $\begin{array}{l}\text { IP } \\
\%\end{array}$ & $\begin{array}{c}\text { CEL } \\
\mathrm{d} / \mathrm{mm} 2\end{array}$ & $\begin{array}{c}\text { EST } \\
\text { d/mm2 }\end{array}$ & $\begin{array}{c}\text { TRI } \\
\mathrm{d} / \mathrm{mm} 2\end{array}$ & $\begin{array}{l}\text { IE } \\
\%\end{array}$ & $\begin{array}{l}\text { IP } \\
\%\end{array}$ \\
\hline Achyrocline satureioides & 1129,31 & 0 & 125,86 & 0 & 10,03 & 1034,48 & 65,52 & 174,48 & 5,96 & 14,43 \\
\hline Acmella decumbens & 644,83 & 84,48 & 0 & 11,58 & 0 & 500 & 84,48 & 0 & 14,45 & 0 \\
\hline $\begin{array}{l}\text { Alternanthera philoxeroides } \\
\text { f. philoxeroides }\end{array}$ & 631,89 & 139,65 & 3,45 & 17,96 & 0,45 & 527,58 & 113,81 & 4,31 & 17,56 & 1,85 \\
\hline Alternanthera pungens & 350 & 101,77 & 0 & 22,52 & 0 & 429,31 & 77,59 & 3,45 & 15,31 & 0,81 \\
\hline $\begin{array}{c}\text { Amaranthus hybridus } \\
\text { ssp. hybridus }\end{array}$ & 1115,51 & 246,55 & 0 & 18,1 & 0 & 1246,55 & 253,45 & 3,45 & 16,9 & 0,27 \\
\hline Ambrosia tenuifolia & 786,2 & 60,34 & 34,48 & 7,13 & 4,2 & 1070,69 & 79,31 & 41,38 & 6,9 & 3,72 \\
\hline Bidens pilosa & 469,79 & 3,45 & 10,34 & 0,72 & 2,15 & 494,83 & 131,03 & 13,79 & 20,94 & 2,71 \\
\hline Borago officinalis & 593,1 & 112,62 & 4,55 & 15,96 & 0,76 & 813,79 & 280,41 & 9,17 & 25,63 & 1,11 \\
\hline Cichorium intybus & 514,27 & 77,59 & 11,17 & 13,1 & 2,12 & 603,45 & 76,69 & 1,72 & 11,27 & 0,28 \\
\hline Commelina erecta & 462,12 & 18,94 & 15,15 & 3,94 & 3,17 & 579,54 & 49,24 & 26,51 & 7,83 & 4,35 \\
\hline Conyza bonariensis & 503,45 & 75,86 & 26,72 & 13,09 & 5,04 & 550 & 73,27 & 23,27 & 11,76 & 4,06 \\
\hline Crassula multicava & 373,21 & 21,87 & 0 & 5,62 & 0 & 511,75 & 59,52 & 0 & 10,68 & 0 \\
\hline Cymbopogon citratus & 693,11 & 37,93 & 3,44 & 5,19 & 0,14 & 1389,65 & 231,03 & 144,83 & 14,25 & 9,44 \\
\hline Dysphania retusa & 867,24 & 175,86 & 48,27 & 16,86 & 5,27 & 803,45 & 213,79 & 55,17 & 21,01 & 6,42 \\
\hline Echium plantagineum & 1492,42 & 390,15 & 26,51 & 20,72 & 1,74 & 1469,69 & 428,03 & 3,79 & 22,55 & 0,26 \\
\hline Euphorbia serpens & 1589,65 & 327,58 & 0 & 17,11 & 0 & 1428,03 & 280,3 & 0 & 16,41 & 0 \\
\hline Fumaria capreolata & 222,41 & 0 & 0 & 0 & 0 & 300 & 80,41 & 0 & 21,14 & 0 \\
\hline Galinsoga parviflora & 341,38 & 0 & 0 & 0 & 0 & 775,86 & 55,17 & 0 & 6,64 & 0 \\
\hline Gamochaeta coarctata & 360,34 & 50 & 43,1 & 12,18 & 10,68 & 356,9 & 36,21 & 25,86 & 9,21 & 6,82 \\
\hline Geranium molle & 1431,03 & 139,65 & 24,14 & 8,89 & 1,66 & 1341,38 & 256,9 & 36,21 & 16,07 & 2,63 \\
\hline Cantinoa mutabilis & 1401,51 & 75,75 & 56,81 & 5,12 & 3,91 & 1647,73 & 450,76 & 83,33 & 21,48 & 4,43 \\
\hline Lepidium bonariense & 667,24 & 205,17 & 0 & 23,52 & 0 & 529,31 & 136,48 & 3,45 & 20,26 & 0,64 \\
\hline Lepidium didymum & 898,27 & 306,9 & 0 & 25,46 & 0 & 986,21 & 301,72 & 0 & 23,43 & 0 \\
\hline Malva parviflora & 1022,72 & 196,97 & 45,45 & 16,14 & 4,25 & 1393,94 & 409,09 & 53,03 & 22,69 & 3,66 \\
\hline Medicago polymorpha & 1618,96 & 410,34 & 0 & 20,22 & 0 & 1518,96 & 400 & 3,51 & 20,84 & 0,25 \\
\hline Melilotus indicus & 848,27 & 200 & 1,72 & 19,08 & 0,2 & 753,45 & 201,72 & 0 & 20,97 & 0 \\
\hline Mentha $x$ piperita & 810,34 & 6,9 & 6,9 & 0,84 & 0,84 & 1458,33 & 348,48 & 1,25 & 19,29 & 1,28 \\
\hline Mikania micrantha & 460,34 & 0 & 9,2 & 0 & 1,48 & 941,29 & 102,27 & 48,01 & 9,21 & 3,58 \\
\hline Modiola carolineana & 787,88 & 121,21 & 30,3 & 13,33 & 3,7 & 1257,57 & 272,73 & 30,3 & 17,82 & 2,35 \\
\hline Ocimum basilicum & 424,24 & 115,53 & 17,04 & 21,4 & 3,86 & 403,41 & 126,89 & 20,83 & 23,9 & 4,91 \\
\hline Oenothera affinis & 1185,6 & 164,7 & 363,64 & 12,2 & 23,47 & 1126,82 & 162,88 & 244,24 & 12,63 & 17,81 \\
\hline
\end{tabular}




\begin{tabular}{|c|c|c|c|c|c|c|c|c|c|c|}
\hline \multirow[b]{2}{*}{ TAXA / CARACTERES } & \multicolumn{3}{|c|}{ EPIDERMIS } & \multicolumn{2}{|c|}{ ADAXIAL } & \multicolumn{3}{|c|}{ EPIDERMIS } & \multicolumn{2}{|c|}{ ABAXIAL } \\
\hline & $\begin{array}{c}\text { CEL } \\
\mathrm{d} / \mathrm{mm} 2\end{array}$ & $\begin{array}{c}\text { EST } \\
\mathrm{d} / \mathrm{mm} 2\end{array}$ & $\begin{array}{c}\text { TRI } \\
\mathrm{d} / \mathrm{mm} 2\end{array}$ & $\begin{array}{l}\text { IE } \\
\%\end{array}$ & $\begin{array}{l}\text { IP } \\
\%\end{array}$ & $\begin{array}{c}\text { CEL } \\
\text { d/mm2 }\end{array}$ & $\begin{array}{c}\text { EST } \\
\text { d/mm2 }\end{array}$ & $\begin{array}{c}\text { TRI } \\
\mathrm{d} / \mathrm{mm} 2\end{array}$ & $\begin{array}{l}\text { IE } \\
\%\end{array}$ & $\begin{array}{l}\text { IP } \\
\%\end{array}$ \\
\hline Origanum vulgare & 1255,68 & 3,79 & 54,92 & 0,31 & 4,19 & 1710,23 & 422,35 & 121,21 & 19,81 & 6,62 \\
\hline $\begin{array}{r}\text { Oxalis articulata } \\
\text { ssp. articulata }\end{array}$ & 412,88 & 0 & 11,36 & 0 & 2,68 & 579,54 & 219,7 & 7,57 & 27,49 & 12,91 \\
\hline Parietaria debilis & 594,83 & 0 & 62,07 & 0 & 9,45 & 1124,14 & 251,72 & 115,52 & 18,29 & 9,32 \\
\hline $\begin{array}{c}\text { Phyla nodiflora } \\
\text { var. minor }\end{array}$ & 1564,39 & 329,54 & 75,75 & 17,4 & 4,61 & 1905,3 & 310,6 & 185,6 & 14,02 & 8,88 \\
\hline Phyllanthus niruri & 1500 & 0 & 0 & 0 & 0 & 1719,7 & 340,91 & 0 & 16,54 & 0 \\
\hline Physalis viscosa & 675,86 & 140,21 & 0 & 17,18 & 0 & 533,79 & 100,69 & 3,51 & 15,87 & 0,25 \\
\hline Plantago lanceolata & 636,78 & 137,93 & 6,9 & 17,81 & 1,07 & 887,36 & 255,17 & 20,69 & 22,33 & 2,28 \\
\hline Plantago major & 381,61 & 80,46 & 4,6 & 17,41 & 1,18 & 439,08 & 117,24 & 1,33 & 21,08 & 2,05 \\
\hline $\begin{array}{c}\text { Plantago tomentosa } \\
\text { ssp. tomentosa }\end{array}$ & 713,79 & 224,14 & 10,34 & 23,9 & 1,43 & 963,79 & 315,51 & 13,79 & 24,66 & 8,98 \\
\hline Pluchea sagittalis & 1198,27 & 77,59 & 31,03 & 6,08 & 2,52 & 1287,93 & 96,55 & 46,55 & 6,97 & 3,49 \\
\hline Portulaca oleracea & 367,42 & 44,51 & 0 & 10,81 & 0 & 373,11 & 56,82 & 0 & 13,22 & 0 \\
\hline Raphanus sativus & 915,52 & 244,83 & 0 & 21,1 & 0 & 1122,41 & 372,41 & 0 & 24,91 & 0 \\
\hline Rosmarinus officinalis & 3075,75 & 0 & 11,36 & 0 & 0,37 & 1484,85 & 803,03 & 30,3 & 35,09 & 2,01 \\
\hline Salpichroa origanifolia & 284,48 & 29,31 & 3,45 & 9,34 & 1,2 & 356,9 & 79,31 & 8,62 & 18,18 & 2,36 \\
\hline Salvia officinalis & 1549,24 & 149,62 & 329,54 & 8,81 & 17,54 & 2155,3 & 471,59 & 259,47 & 17,95 & 10,74 \\
\hline Sida rhombifolia & 2674,24 & 621,21 & 15,15 & 18,85 & 0,56 & 3246,21 & 1556,82 & 331,36 & 32,41 & 9,26 \\
\hline $\begin{array}{l}\text { Sisyrinchium chilense } \\
\text { var. chilense }\end{array}$ & 333,33 & 0 & 0 & 0 & 0 & 330,3 & 154,54 & 0 & 31,87 & 0 \\
\hline Solanum sisymbriifolium & 1210,23 & 242,42 & 32,21 & 16,69 & 2,59 & 1901,51 & 446,97 & 22,73 & 19,03 & 1,18 \\
\hline $\begin{array}{c}\text { Solidago chilensis } \\
\text { var. chilensis }\end{array}$ & 1333,62 & 69,83 & 22,41 & 4,97 & 1,65 & 1312,07 & 71,55 & 20,69 & 5,17 & 1,55 \\
\hline Sonchus oleraceus & 705,17 & 132,75 & 0 & 15,84 & 0 & 565,52 & 124,14 & 0 & 18 & 0 \\
\hline Taraxacum officinale & 874,14 & 56,9 & 0 & 6,11 & 0 & 1027,59 & 65,52 & 0 & 5,99 & 0 \\
\hline Trifolium repens & 1191,38 & 218,96 & 1,72 & 15,52 & 0,14 & 802,75 & 106,21 & 4,14 & 11,68 & 0,51 \\
\hline Tripogandra diuretica & 335,23 & 0 & 9,47 & 0 & 2,75 & 691,29 & 75,75 & 17,04 & 9,88 & 2,41 \\
\hline Urtica urens & 424,14 & 0 & 37,93 & 0 & 8,21 & 989,65 & 272,41 & 62,07 & 21,51 & 5,91 \\
\hline Verbascum virgatum & 322,41 & 27,59 & 5,17 & 7,88 & 1,58 & 555,86 & 169,65 & 9,65 & 23,38 & 1,71 \\
\hline $\begin{array}{r}\text { Verbena bonariensis } \\
\text { var. bonariensis }\end{array}$ & 1109,85 & 0 & 147,72 & 0 & 11,75 & 3303,03 & 616,64 & 284,09 & 15,67 & 7,92 \\
\hline $\begin{array}{r}\text { Verbena litoralis } \\
\text { var. litoralis }\end{array}$ & 1164,77 & 128,78 & 64,39 & 9,97 & 5,18 & 1884,47 & 526,51 & 62,5 & 21,79 & 3,28 \\
\hline Verbena montevidensis & 969,7 & 56,82 & 53,03 & 5,53 & 5,18 & 1882,57 & 439,39 & 87,12 & 18,92 & 4,42 \\
\hline Vinca major & 305,17 & 0 & 0 & 0 & 0 & 572,41 & 92,41 & 0 & 13,91 & 0 \\
\hline Xanthium spinosum & 1018,96 & 87,93 & 82,76 & 7,94 & 7,51 & 1227,27 & 325,72 & 159,09 & 20,97 & 11,47 \\
\hline Xanthium strumarium & 1686,21 & 101,72 & 22,41 & 5,69 & 1,31 & 2089,65 & 289,65 & 10,34 & 12,17 & 0,49 \\
\hline
\end{tabular}




\section{Conclusión}

Se estudió la hoja que es el órgano más frecuentemente utilizado en la medicina popular y se amplió el conocimiento botánico de las especies incluidas en el estudio. Los datos cualitativos y parámetros micrográficos obtenidos han permitido elaborar una clave dicotómica aplicable en el control de calidad botánica.

\section{Agradecimientos}

A los curadores de los herbarios por facilitar el material vegetal. A Gloria Barboza por su gentil contribución con material bibliográfico. A los revisores por la lectura crítica del manuscrito y sugerencias brindadas que ayudaron a mejorar el mismo. A la Secretaría de Ciencia y Técnica de la Universidad Nacional de La Plata por el financiamiento económico de los estudios.

\section{Bibliografía}

ALANIZ, F.S., L. N. CARIDDI, M. GIORDANA, D. CALVO, L. GONZÁlEZ PEREYRA, M. L. GAMBERO, M. S. DEMO \& A. M. MALDONADO. 2010. Efectos antimicrobianos, antifúngico, antialérgicos e inmunomoduladores de Achyrocline satureioides (Asteraceae). X Simposio Argentino. XII Simposio Latinoamericano de Farmacobotánica. Pág. 105.

ALDHEBIANI, A. \& S. JURY. 2013. Anatomical studies on the genus Euphorbia L. Saudi Arabia (Subgenera: Triucalli, Ermophyton, Esula and Chamaesyce). International Research Journal of Plant Science 4: 168-191.

ALONSO, J. R. \& C. DESMARCHELIER. 2005. Plantas medicinales autóctonas de la Argentina. Bases Científicas para su Aplicación en Atención Primaria de la salud. L.O.L.A. (Literature of Latin America), Buenos Aires. 663 pp.

AMORIN, J. \& E. N. ORFILA. 1972. Xanthium spinosum L. maleza utilizada en la medicina popular argentina. Rev. Fac. Agronomía (3ra. Época) 48: 155-169.

AMORIN, M., J. P. DE PAULA, R. Z. DA SILVA, P. V. FARAGO \& J. M. BUDEL. 2014. Pharmacobotanical study of the leaf and stem of Mikania lanuginosa for its quality control. Rev. Bras. Farmacogn. 24: 531537.
ANCIBOR, E. 1992. Anatomía ecológica de la vegetación de La Puna de Mendoza. I. Anatomía foliar. Parodiana 7: 63-76.

APÓSTOLO, N. M. 2005. Caracteres anatómicos de la vegetación costera del Río Salado (Noroeste de la provincia de Buenos Aires, Argentina). Bol. Soc. Argent. Bot. 40: 215-227.

ARAMBARRI, A. M., M. C. NOVOA, M. P. HERNÁNDEZ, M. N. COLARES \& V. G. PERROTTA. 2013. Anatomía de Dicotiledóneas: tallos de hierbas terrestres medicinales rioplatenses (Buenos Aires, Argentina). Bonplandia 22: 5-28.

BACIGALUPO, N. M. \& J. A. HURRELL. 2008. Flora rioplatense: sistemática, ecología y etnobotánica de las plantas vasculares rioplatenses en: (HURRELL, J. A., G. DELUCCHI \& M. CORREA), 1ra ed., L.O.L.A., Buenos Aires, V1, parte 3. Monocotiledóneas. Alismatales, Arecales, Commelinales, Zingiberales: 336 pp.

BARBOZA, G. E., J. J. CANTERO, C. O. NÚÑEZ \& L. ARIZA ESPINAR. 2006. Flora medicinal de la provincia de Córdoba (Argentina), Museo Botánico de Córdoba, Gráficamente ediciones, Córdoba. 1264 pp.

BARBOZA, G. E., J. J. CANTERO, C. O. NÚÑEZ, A. PACCIARONI \& L. ARIZA ESPINAR. 2009. Medicinal Plants: A general review and a phytochemical and ethnopharmacological screening of the native Argentine Flora. Kurtziana 34 : 7-365.

BARBOZA, G. E., N. BONZANI, E. M. FILIPPA, M. C. LUJÁN, R. MORERO, M. BUGATTI, N. DECOLATTI \& L. ARIZA ESPINAR. 2001. Atlas histo-morfológico de plantas de interés medicinal de uso corriente en Argentina. Museo Botánico de Córdoba, Ser. Especial 1, 212 pp.

BAYÓN, N. D., M. N. COLARES, A. M. ARAMBARRI \& S. VIÑA. 2000. Anatomía de las especies medicinales de la Provincia Pampeana: Plantago L. (Plantaginaceae). Acta Farm. Bonaerense 19: 263-272.

BOHM, B. C., C. L. GONÇALVEX, L. F. D. SCHUCH, A. B. P. CHAFFE, R. N. SCHUBERT, G. SCHIEDECK \& A. FACCIN. 2012. XXII Simpósio de Plantas Medicinais do Brasil. 18 al 21 setembro. Bento Gonçalves, Brasil.

BONZANI, N. E., E. M. FILIPPA \& G. E. BARBOZA. 1997. Particularidades epidérmicas en algunas especies de Verbenaceae. Anales Inst. Biol. Univ. Nac. Mexico 68: 47-56.

BONZANI, N. E., G. E. BARBOZA, M. A. BUGATTI \& L. ARIZA ESPINAR. 2003. Morpho-histological studies in the aromatic species of Chenopodium from Argentina. Fitoterapia 74: 207-225.

BURKART, A. 1957. Ojeada sinóptica sobre la vegetación del Delta del río Paraná. Darwiniana 11: 475-563.

CABRERA, A. L. 1949. Las comunidades vegetales de los alrededores de La Plata Provincia de Buenos Aires, República Argentina. Lilloa 20: 269-274. 
CABRERA, A. L. 1963. Flora de la provincia de Buenos Aires. Vol.4, parte 6, Colección Científ. INTA. 443 pp.

CABRERA, A. L \& G. DAWSON. 1944. La selva marginal de Punta Lara en la ribera argentina del Río de La Plata. Revista Museo de La Plata, Secc. Bot. 5: 267-382.

CABRERA, A. L \& A. BURKART. 1974. Compositae. En A. Burkart, (dir.), Flora ilustrada de Entre Ríos (Argentina), Colecc. Científ. INTA 6: 107-110.

CABRERA, A. L \& E. M. ZARDINI. 1978. Manual de la flora de los alrededores de Buenos Aires, 2 ed., Ed. Acme, Buenos Aires. 755 pp.

CAMBI, V., A. BUCCIARELLI, A. FLEMMER \& P. HANSEN. 2006. Morfoanatomía de Pluchea sagittalis (Asteraceae), especie nativa de interés medicinal. Acta Farm. Bonaerense 25: 43-49.

COLARES, M. 2011. Mikania cordifolia y Mikania micrantha (Asteraceae): especies medicinales nativas de las reservas naturales de Punta Lara e Isla Martín García, Buenos Aires, Argentina. Tesis de Magister en Plantas Medicinales, Facultad de Ciencias Exactas, SEDICI, UNLP.

COLARES, M., N. D. BAYÓN, S. A. STENGLEIN \& A. M. ARAMBARRI. 1999. Anatomía y etnobotánica de las especies medicinales de la provincia pampeana: Solanaceae (excepto Grabowskia y Solanum). Acta Farm. Bonaerense 18: 171-182.

COLARES, M., A. MUGUERZA, M. A. ROSELlA \& A. E. CONSOLINI. 2013. Antispasmodic effects of Mikania micrantha Kunth and dual intestinal effect of Mikania cordifolia (L. f.)Willd (Asteraceae) on isolated rat thin intestine. Pharmacologyonline 2: $1-11$.

COLARES, M., M. P. HERNÁNDEZ, M. C. NOVOA, V. G. PERROTTA, S. AUGUET \& A. M. ARAMBARRI. 2014. Anatomía comparada de raíces medicinales de hierbas terrestres rioplatenses (Buenos Aires, República Argentina). Dominguezia 30: 5-18.

DA SILVA FILHO, F. A., P. L. DE OLIVEIRA \& J. E. DE ARAUJO MARIATH. 1992. Tricomas de Chenopodium retusum Juss. ex Moq. (Chenopodiaceae). Insula, Florianópolis 21: 43-58.

DEGEN, R. Y. GONZÁLEZ, L. OAKLEY \& G. DELMAS. 2011. Estudio morfo-anatómico de especies del género Phyllanthus L. (Phyllanthaceae) comercializadas como "para para'i" en Paraguay. XXXIII Jornadas Argentinas de Botánica. Boletín de la Sociedad Argentina de Botánica 46: 92-93.

DIORIO, L. A. 1986. Anatomía foliar de seis especies argentinas del género Lepidium (Cruciferae). Parodiana 4: 1-16.

DOMÍNGUEZ, J. A. 1928. Contribuciones a la materia médica argentina. Instituto de Botánica y Farmacología (Facultad de Ciencias Médicas de Buenos Aires), Ed. Peuser, Buenos Aires. 433 pp.
DOS SANTOS, A. L., V. F. PÉRES, D. J. MOURA, R. A. JACQUES, E. B. CARAMÃO \& P. PAUWELZ. 2012. XXII Simposio de Plantas Medicinais do Brasil, 18 al 21 de setembro. Bento Gonçalves, Brasil.

DUARTE, M. R. \& M. C. DEBUR. 2004. Characters of the leaf and stem morpho-anatomy of Alternanthera brasiliana (L.) O. Kuntze, Amaranthaceae. Braz. J. Pharm. Sci. 40: 85-92.

ELB, P. M., G. F. MELO-DE PINNA \& N. L. DE MENEZES. 2010. Morphology and anatomy of leaf miners in two species of Commelinaceae (Commelina diffusa Burm. f. and Floscopa glabrata (Kunth) Hassk). Acta Bot. Bras. 24: 283-287.

FIGUEROA, S. D., N. DOTTORI \& M. T. COSA. 2011. Anatomía vegetativa de Portulaca oleracea (Portulacaceae) especie de interés medicinal. XXXIII Jornadas Argentinas de Botánica, Bol. Soc. Argent. Bot. 46(supl.): 95.

FIGUEROA, S. D., M. T. COSA \& N. DOTTORI. 2013. Anatomía foliar de Portulaca oleracea, $P$. cryptapetala (Portulacaceae) y Talinum paniculatum (Talinaceae). XXXIV Jornadas Argentinas de Botánica, Bol. Soc. Argent. Bot. 48(supl.): 141.

FILIPPA, E., G. E. BARBOZA, M. C. LUJÁN \& L. ARIZA ESPINAR. 1999. Anatomía foliar de las especies centro-argentinas de Plantago (Plantaginaceae). Darwiniana 37: 1-13.

FILIPOV, A. 1997. La farmacopea natural en los sistemas terapéuticos de los indígenas pilagá. Parodiana 10: 35-74.

FREIRE, S. E., A. M. ARAMBARRI, N. D. BAYÓN, G. SANCHO, E. URTUBEY, C. MONTI, M. C. NOVOA \& M. N. COLARES. 2005. Epidermal characteristics of toxic plants for cattle from the Salado river basin (Buenos Aires, Argentina). Bol. Soc. Argent. Bot. 40: 241-281.

FRYNS-CLAESSENS, E. \& W. VAN COTTHEM. 1973. A new classification of the ontogenetic types of stomata. Bot. Rev. 39: 85-87.

GATTUSO, S. J. \& M. A. GATTUSO. 1998. Caracteres anatómicos y exomorfológicos distintivos de Achyrocline satureioides (Lam.) DC. (AsteraceaeInuleae). Acta Farm. Bonaerense 17: 255-261.

GIBERTI, G. C. 1981. Las malvas y las salvias de la medicina popular del noroeste argentino. Parodiana 1: 31-96.

HADID, M. A. PÉREZ, N. DOTTORI \& M. T. COSA. 2011. Anatomía de la hoja de Oxalis articulata (Oxalidaceae) y su relación con los movimientos násticos. XXXIII Jornadas Argentinas de Botánica, Misiones Bol. Soc. Argent. Bot. 46(supl.): 100.

HAUMAN, L. 1925. La vegetation de l'lle Martin Garcia dans le Rio de La Plata, Publicación del Instituto de Investigación Geográfica, Facultad de Filosofía y Letras, Universidad de Buenos Aires. 39 pp. 
HERNÁNDEZ, M. \& P. ALBORNOZ. 2001. Morfología y anatomía foliar en especies de Cheilanthes (Pteridaceae) del noroeste argentino. Lilloa 40: 187-207.

HERNÁNDEZ, M. P., M. COLARES \& S. M. CIVITELLA. 2009. Plantas utilizadas en medicina popular en un sector del Partido de Berisso, Buenos Aires, Argentina. Bol. Latinoam. Caribe Plant Med. Aromat. 8: 435-444.

HERNÁNDEZ, M. P., S. M. CIVITELlA \& V. G. ROSATO. 2010. Uso medicinal popular de plantas y líquenes de la Isla Paulino, Provincia de Buenos Aires, Argentina. Bol. Latinoam. Caribe Plant Med. Aromat. 9: 258-268.

HERNÁNDEZ, M. P., M. C. NOVOA, S. M. CIVITELLA, D. MASON \& A. OVIEDO. 2013. Plantas usadas en medicina popular en la Isla Santiago, Buenos Aires, Argentina. Bol. Latinoam. Caribe Plant Med. Aromat. 12: 385-399.

HIERONYMUS, J. 1882. Plantae diaphoricae florae argentinae. Bol. Acad. Cienc. Córdoba 4: 199-598.

HURRELL, J. A., D. H. BAZZANO \& G. DELUCCHI. 2006. Dicotiledóneas herbáceas 1. Nativas y exóticas. Biota Rioplatense XI. L.O.L.A., Buenos Aires. 288pp.

HURRELL, J. A., D. H. BAZZANO \& G. DELUCCHI. 2007. Dicotiledóneas herbáceas 2. Nativas y exóticas. Biota Rioplatense XI. L.O.L.A., Buenos Aires. 288pp.

HURRELL, J. A., E. A. ULIBARRI, P. M. ARENAS \& M. L. POCHETTINO. 2011a. Plantas de herboristería. L.O.L.A., Buenos Aires. 242 pp.

HURRELL, J. A., E. A. ULIBARRI, J. P. PUENTES, F. BUET COSTANTINO, P. M. ARENAS \& M. L. POCHETTINO. 2011b. Leguminosas medicinales y alimenticias utilizadas en la conurbación Buenos Aires-La Plata, Argentina. Bol. Latinoam. Caribe Plant. Med. Aromat. 10: 443-445.

JOHANSEN, D. A. 1940. Plant microtechnique. McGraw- Hill Book Company, New York.

KORWAR, P. G., A. K. BEKNAL, B. S. PATIL, M. A. HALKAI, U. KULKARNI, R. C. HARIPRASANNA \& S. R. SOODAM. 2010. A study on phytochemical investigation of Drymaria quercifolia Linn rhizome. Int. J. Pharm. Sci. Res. (IJPSR) 1: 148-158.

KUTSCHKER, A., H. MENOYO \& V. HECHEM. 2007. Plantas medicinales de uso popular en comunidades del oeste del Chubut. Estación Experimental Agropecuaria de Esquel, Chubut. 139 pp.

LAHITTE, H. B. \& J. A. HURRELL. 1996. Las plantas de la medicina popular de la isla Martín García. Comisión de Investigaciones Científicas de la provincia de Buenos Aires (CICPBA). 246 pp.

LAHITTE, H. B. \& J. A. HURRELL, K. MEHLTRETER, M. J. BELGRANO, L. S. JANKOWSKI, M. P. HALOUA \& G. CANDA. 1997. Plantas de la costa. L.O.L.A., Buenos Aires. 200 pp.
LAHitTE, H. B. \& J. A. HURRELL, M. J. BELGRANO, L. S. JANKOWSKI, P. HALOUA \& K. MEHLTRETER. 2004. Plantas medicinales rioplatenses. L.O.L.A., Buenos Aires. 240 pp.

LUJÁN, M. C. \& G. E. BARBOZA. 1999. Contribution to the study of some Argentinian medicinal plants and comercial quality control. Acta Horticulturae 503: 141-154.

LUJÁN, M. C., N. BONZANI, E. FILIPPA \& G. E. BARBOZA. 2004. La "fumarias" (Fumaria spp., Fumariaceae) de uso etnomédico en Argentina y sus adulterantes. Acta Farm. Bonaerense 23: 154-164.

MANDRILE, E. L. 2003. Farmacognosia. Plantas medicinales que se dispensan en Argentina. 1. Colegio de Farmacéuticos de la Provincia de Buenos Aires. Impresos Gráfica Dasa S.A., 289 pp.

MARTÍNEZ CROVETTO, R. 1964. Estudios etnobotánicos I. Nombres de plantas y su utilidad según los indios tobas del este del Chaco. Bonplandia 1: 279-333.

MARTÍNEZ CROVETTO, R. 1981a. Las plantas utilizadas en medicina popular en el noroeste de Corrientes (República Argentina). Miscelánea 69: 1-139.

MARTÍNEZ CROVETTO, R. 1981b. Plantas reguladoras de la fecundidad utilizadas en la medicina popular del nordeste argentino. Parodiana 1: 97-118.

MARRASSINI, C., S. GORZALCZANY \& G. FERRARO. 2010. Actividad analgésica de dos especies de Urtica con usos etnomédicos en la República Argentina. Dominguezia 26: 21-29.

METCALFE, C. R. \& L. CHALK. 1950. Anatomy of the Dicotyledons: leaves, stem, and wood in relation to taxonomy with notes on economic uses. Vol. 1, 2. Clarendon Press, Oxford. 1500 pp.

METCALFE, C. R. \& L. CHALK. 1979. Anatomy of the Dicotyledons. Vol. 1. Clarendon Press, Oxford. $276 \mathrm{pp}$.

MONTI, C., M. C. NOVOA \& C. E. VIZCAÍNO. 2003. Anatomía y etnobotánica de dos especies de Boraginaceae de la provincia pampeana (Argentina) usadas en la medicina popular. Acta Farm. Bonaerense 22: 197-201.

NOVOA, M. C., M. N. COLARES \& A. M. ARAMBARRI. 2012. Anatomy of monocotyledons: stems and rhizomes of land herbs used as medicinal in the Río de La Plata area (Argentina). Bonplandia 21: $53-61$.

NUGHES, L., M. COLARES, M. HERNÁNDEZ \& A. ARAMBARRI. 2013. Morfo-anatomía de las hojas de Celtis ehrenbergiana (Celtidaceae) desarrolladas bajo condiciones naturales de sol y sombra. Bonplandia 22: 47-58.

ORFILA, E.N. \& C. D’ALFONSO. 1999. Catálogo preliminar de la flora medicinal serrana de Azul (Buenos Aires, Rep. Arg.). Dominguezia 15: 27-38. 
PEREZ, B., G. E. YORMANN, M. COSTAGUTA \& N. M. APÓSTOLO. 2013. Anatomía de hoja y tallo de Conyza bonariensis (L.) Cronquist (Asteraceae): una especie medicinal de la provincia de Buenos Aires. XXXIV Jornadas Argentinas de Botánica, Bol. Soc. Argent. Bot. 48(supl.): 151.

POCHETTINO, M. L., M. R. MARTÍNEZ, B. ITTEN \& M. ZUCARO. 1997. Las plantas medicinales como recurso terapéutico en una población urbana: estudio etnobotánico en Hernández (Pdo. La Plata, Prov. Buenos Aires, Argentina). Parodiana 10: 141-152.

QUAGLIO, A. E. V., L. D. ALMEIDA JUNIOR, A. S. CHAGAS, A. TANIMOTO, A. WITAICENIS, C. A. R. A. COSTA \& L. C. DI STASI. 2012. XXII Simpósio de Plantas Medicinais do Brasil. 18 al 21 setembro. Bento Gonçalves, Brasil.

ROITMANG. \& J.A.HURRELL. 2009. Flora Rioplatense: sistemática, ecología y etnobotánica de las plantas vasculares rioplatenses en: (HURRELL, J. A., G. DELUCCHI \& M. CORREA, 1ra ed., L.O.L.A., Buenos Aires, V4, parte 3. Monocotiledóneas. Asparagales, Dioscoreales, Liliales: 424 pp.

ROMERO JIMÉNEZ, M, E. OLAZÁBAL MANSO, Y. MARTÍNEZ, H. SERRANO \& A. MONTEAGUDO. 2002. Actividad fasciolicida in vitro de Portulaca oleracea L. Acta Farm. Bonaerense 21: 297-300.

RONDINA, R. V. D., A. L. BANDONI \& J. D. COUSIO. 2008. Especies medicinales argentinas con potencial actividad analgésica. Dominguezia 24: 47-69.

RONDINA, R. V. D., A. L. BANDONI \& J. D. COUSIO. 2010. Argentine medicinal plants with potential antifungal activity. Dominguezia 26: 31-39.

RUZIN, S. E. 1999. Plant microtechnique and microscopy. University Press, Oxford. 323 pp.

SALISBURY, E. 1927. On the causes and ecological significance ofstomatal frequency with special reference to the woodland flora. Phil. Trans. R. Soc. Lond., Ser B, 216: 1-65.

SÁNCHEZ GOVÍN, E. I. M. LEAL LÓPEZ, L. FUENTES HERNÁNDEZ \& C. A. RODRÍGUEZ FERRADA. 2000. Estudio farmacognóstico de Ocimum basilicum L. (albahaca blanca) Rev. Cubana Farm. 34: 187-195.

SIMON, L. E. 1987. Morfología, distribución y valor diagnóstico de los pelos glandulares en especies de Chenopodium L. (Chenopodiaceae). Notas Mus. La Plata (Bot.) 21: 99-110.
STACE, C. A. 1965. Cuticular studies as an aid to plant anatomy. Bull. Br. Mus. (Nat. Hist.) Bot. 4: 1-78.

STENGLEIN, S. A. 2001. Características epidérmicas de la hoja de especies medicinales del género Solanum L. (Solanaceae) de la Provincia Biogeográfica Pampeana. Acta Farm. Bonaerense 20: 265-274.

STIEFKENS, L., M. HADID, A. PÉREZ, N. DOTTORI \& M. T. COSA. 2010. Anatomía vegetative en tres especies de Salpichroa (Solanaceae). X Simposio Argentino y XIII Simposio Latinoamericano de Farmacobotánica. Libro de resúmenes, pág. 77. Facultad de Ciencias Químicas, IMBIVCONICET, Universidad Nacional de Córdoba.

TOURSARKISSIAN, M. 1980. Plantas medicinales de la Argentina. Sus nombres botánicos vulgares, usos y distribución geográfica. Ed. Hemisferio Sur, Buenos Aires. 168 pp.

ULIBARRI, E. A., E. V. GÓMEZ SOSA, A. M. CIALDELLA, R. FORTUNATO \& D. BAZZANO. 2002. Leguminosas nativas y exóticas. En: Hurrell, J. A. \& H. B. Lahitte (eds.). Biota Rioplatense VII. L.O.L.A., Buenos Aires. 320 pp.

VIZCAÍNO, C.E., M. C. NOVOA \& M. N. COLARES \& G. DELUCCHI. 1998. Anatomía y etnobotánica de las especies medicinales de Monocotiledóneas de la Estepa Pampeana Argentina: Cannaceae, Commelinaceae e Iridaceae. Acta Farm. Bonaerense 17: 85-90.

YAGUEDDÚ, C. \& M. S. CID. 1992. Caracteres epidérmicos de Dicotiledóneas de la pampa deprimida bonaerense, de utilidad en microanálisis de dietas. Rev. Arg. Prod. Anim. 12: 265-279.

YAGUEDDÚ, C., V. COMPARATORE \& G. PAOLETTI. 2009. Identification of six Papilionaceae species by epidermal characteristics: microanalysis of hand-composed mixtures. Bol. Soc. Argent. Bot. 44: 305-315.

ZARLAVSKY, G. E. 2014. Histología vegetal. Técnicas simples y complejas. Sociedad Argentina de Botánica. 198 pp.

ZULOAGA, F. O., O. MORRONE \& M. J. BELGRANO. 2008. Catálogo de las plantas vasculares del Cono Sur (Argentina, Sur de Brasil, Chile, Paraguay y Uruguay). I. Monogr. Syst. Bot. Missouri Bot. Gard. 107: 983 pp. http://www. darwin.edu.ar/ consultado: 12.02.2015.

Original recibido el 14 de abril de 2015; aceptado el 1 de julio de 2015. 
Apéndice 1. Familias y especies estudiadas en orden alfabético por sus nombres científicos, nombre vulgar de uso frecuente, uso popular y actividad biológica.

AMARANTHACEAE: Alternanthera philoxeroides f. philoxeroides "vinagrillo". Usos: digestivo, diurético, hepático (Hieronymus, 1882; Lahitte \& Hurrell, 1996). Actividad biológica: antitumoral y antiviral (Barboza et al., 2009). Alternanthera pungens "yerba del pollo". Usos: antidermatósico, antisifilítico, depurativo, digestivo, diurético, emoliente, estomacal (Hieronymus, 1882; Pochettino et al., 1997; Lahitte et al., 2004). Actividad biológica: antiinflamatorio, diurético, laxante (Alonso \& Desmarchelier, 2005; Barboza et al., 2009). Amaranthus hybridus ssp. hybridus "yuyo colorado". Usos: antiblenorrágico, digestivo, diurético, hepático (Toursarkissian, 1980; Lahitte et al., 2004). Actividad biológica: antianémico; antiespasmódico (Alonso \& Desmarchelier, 2005; Barboza et al., 2006). APOCYNACEAE: Vinca major "vinca". Usos: antiinflamatorio, antineoplásico, en aplicación externa estimula la circulación sanguínea, galactófugo; vulnerario (Lahitte \& Hurrell (1996; Lahitte et al., 2004; Hurrell et al., 2006). ASTERACEAE: Achyrocline satureioides "marcela". Usos: adelgazante, antiasmático, antiespasmódico, eupéptico, expectorante (Martínez Crovetto, 1981a; Orfila \& D’Alfonso, 1999; Lahitte et al., 2004; Hurrell et al., 2011a). Actividad biológica: analgésico, antiespasmódico, antiherpético, antiinflamatorio, antimicrobiano, antioxidante, antiulcerante, antiviral, citotóxico, colagogo, colerético, contra el carcinoma de hígado, hepatoprotector, hipoglucemiante, hipotensor, inmunoestimulante, inmunomodulador, relajante (Alonso \& Desmarchelier, 2005; Barboza et al., 2006, 2009; Alaníz et al., 2010). Acmella decumbens "spilanto". Usos: analgésico de encías y caries, pectoral (Lahitte et al., 2004). Actividad biológica: analgésico, anestésico; antidiarreico; antiinflamatorio, antimicrobiano, antiespasmódico, astringente; cicatrizante, en odontalgias; repelente de insectos (Alonso \& Desmarchelier, 2005; Barboza et al., 2009). Ambrosia tenuifolia "altamisa". Usos: anticonceptivo, antineurálgico, antipediculósico, antipirético, antirreumático, carminativo, contra calambres y contracturas musculares, digestivo (Martínez Crovetto, 1981a; Rondina et al., 2008; Hernández et al., 2013). Actividad biológica de la parte aérea: antibacteriano, antihelmíntico, antiinflamatorio, antiviral, citotóxico (Barboza et al., 2009). Bidens pilosa "amor seco". Usos: abortivo, antiictérico, antiinflamatorio, refrescante (Martínez Crovetto, 1981b; Lahitte et al., 2004; Rondina et al., 2008; Quaglio et al., 2012). Actividad biológica: antibacteriano, antiinflamatorio, antileucémico, antimalárico, antimicrobiano, antiulceroso, diurético, gástrico, hepático, hipoglucemiante, hipotensor (Alonso \& Desmarchelier, 2005; Barboza et al., 2009). Cichorium intybus “achicoria silvestre". Usos: analgésico muscular, antiasmático, antidiarreico, antihelmíntico, antimalárico, aperitivo, colagogo, colerético, diurético, hepático, laxante, oftálmico, refrescante, sudorífico, tónico (Mandrile, 2003; Lahitte et al., 2004; Rondina et al., 2008; Hurrell et al., 2011a). Conyza bonariensis "yerba carnicera". Usos: antiblenorrágico, antiulceroso, cicatrizante, diurético, hepático, para lavar granos, pectoral (Martínez Crovetto, 1964; Lahitte et al., 2004; Hurrell et al., 2011a; Pérez et al., 2013); Actividad biológica: antiespasmódico, antiinflamatorio, antimicrobiano, aperitivo, ansiolítico-sedativo, antitusivo, béquico, cardiotónico, colagogo, colerético, digestivo, diurético, eupéptico, hipotensor (Alonso \& Desmarchelier, 2005; Barboza et al., 2009). Galinsoga parviflora "albahaca silvestre". Usos: antiescorbútico, antipirético, hepático, vulnerario (Hieronymus, 1882; Barboza et al., 2006). Actividad biológica: hipotensivo, hipotérmico (Barboza et al., 2009). Gamochaeta coarctata "vira vira". Usos: analgésico, antipirético, cicatrizante, tónico contra la angustia, cansansio, sarampión y viruela (Lahitte \& Hurrell, 1996; Lahitte et al., 2004). Mikania micrantha "bejuco". Usos: antiespasmódico, antireumático, broncodilatador, contra dolores musculares, expectorante (Lahitte \& Hurrell, 1996; Colares et al., 2013); para dolores musculares y reumáticos (Lahitte \& Hurrell, 1996). Actividad biológica: antibacteriano y tripanosómico (Barboza et al., 2009). Pluchea sagittalis "yerba del lucero". Usos: antiespasmódico, antioxidante, antiséptico, aperitivo, carminativo, colagogo, digestivo, estimulante, resolutivo, vulnerario (Mandrile, 2003; Cambi et al., 2006; Hurrell et al., 2011a). Actividad biológica: antiinflamatorio, antimicrobiano, antioxidante, antipirético, carminativo, colagogo, tónico (Alonso \& Desmarchelier, 2005; Barboza et al., 2009). Solidago chilensis var. chilensis "vara de oro". Usos: anticefalálgico, digestivo, diurético, sedativo (Lahitte \& Hurrell, 1996; Filipov, 1997; Lahitte et al., 2004). Actividad biológica: antídoto de venenos, antiespasmódico, antimicrobiano, antioxidante, antiulceroso, gástrico, repelente de 
insectos (Alonso \& Desmarchelier, 2005; Barboza et al., 2009). Sonchus oleraceus "cerraja". Usos: antinefrítico, antitusivo, antiulceroso de várices, aperitivo, dermatopático, diurético, hepático, laxante (Lahitte et al., 2004; Barboza et al., 2006). Actividad biológica: antitumoral (Barboza et al., 2006). Taraxacum officinale "diente de león". Usos: antidermatósico, antidiabético, antihemorroidal, antiictérico, antirreumático, aperitivo, colagogo, colerético, depurativo, diurético, hipoglucemiante, hipotensor, laxante, provitamínico, tónico (Lahitte \& Hurrell, 1996; Mandrile, 2003; Lahitte et al., 2004; Barboza et al., 2006; Hurrell et al., 2011a; Hernández et al., 2013). Actividad biológica: analgésico, antibacteriano, antifúngico, antiinflamatorio, antioxidante, antitumoral, citotóxico, diurético, hipoglucémico, (Barboza et al., 2006). Xanthium spinosum "cepa caballo". Usos: anticefalálgico, anticonceptivo, antiespasmódico, antilítico, antimalárico, antimicrobiano, antipirético, antirreumático, antivenéreo, colagogo, diurético; emoliente, hepático, laxante, purgante, vulnerario (Amorín \& Orfila, 1972; Lahitte \& Hurrell, 1996; Cambi et al. 2006; Hurrell et al., 2011a). Actividad biológica: antiinflamatorio, colagogo, colerético, contra la leucemia, diurético, hipoglucemiante, laxante (Alonso \& Desmarchelier, 2005; Barboza et al., 2006, 2009). Xanthium strumarium "abrojo grande". Usos: antiespasmódico, antinefrítico, antiséptico, contra afecciones pulmonares, hepático, para tratamientos dérmicos de forúnculos, granos, erupciones cutáneas, vulnerario (Pochettino et al., 1997; Barboza et al., 2006; Hernández et al., 2013). Actividad biológica: antimicrobiano, antiulceroso, gastroprotector (Barboza et al., 2009). BORAGINACEAE: Borago officinalis "borraja". Usos: antiasmático, antiinflamatorio, antipirético, antirreumático, astringente, bronquiolítico, contra la gota, contra el sarampión, diurético, emenagogo, pectoral, sudorífico (Hieronymus, 1882; Mandrile, 2003). Echium plantagineum "flor morada". Usos: antidermatósico, analgésico estomacal, diaforético, diurético, emoliente, vulnerario (Hieronymus, 1882; Lahitte \& Hurrell, 1996; Lahitte et al., 2004; Hurrell et al., 2006; Rondina et al., 2008). Actividad biológica: hepatotóxico y neurotóxico (Barboza et al., 2006). BRASSICACEAE: Lepidium bonariense "mastuerzo de Castilla". Usos: para activar la circulación sanguínea (Martínez Crovetto, 1981a; Lahitte \& Hurrell, 1996; Lahitte et al., 2004). Actividad biológica: antimicrobiano (Barboza et al., 2009). Lepidium didymum "mastuerzo". Usos: anticatarral, antiinflamatorio, antihemorroidal, antineurítico, antipirético, antitumoral, astringente, colerético, digestivo, diurético, emenagogo, estimulante, hemostático, hepático, hipoglucemiante, vulnerario (Martínez Crovetto, 1981a; Pochettino et al., 1997; Hurrell et al., 2007; Hurrell et al., 2011a). Actividad biológica: antialérgico, antianémico, antiinflamatorio, antimicrobiano, antioxidante, aperitivo, colerético, digestivo, hepatoprotector, inmunoestimulante (Alonso \& Desmarchelier, 2005; Barboza et al., 2009). Raphanus sativus "rabanito". Usos: antiartrítico, antinefrítico, aperitivo, hepático (Amorín \& Orfila, 1972; Lahitte \& Hurrell, 1996). CHENOPODIACEAE: Dysphania retusa "paico". Usos: aperitivo, carminativo, digestivo, emenagogo, sudorífico, tónico (Bonzani et al. 2003; Lahitte et al., 2004; Hernández et al., 2009). Como las restantes especies de Dysphania conocidas como "paico" y consumidas principalmente como deben usarse con precaución para evitar el efecto tóxico. COMMELINACEAE: Commelina erecta "Flor de Santa Lucía". Usos: antidermatósico, antipruritoso, contra la conjuntivitis, eritemas, hemorragias oculares y herpes, diurético (Lahitte et al., 2004; Bacigalupo \& Hurrell, 2008; Barboza et al., 2009). Actividad biológica: antimicrobiano, descongestivo ocular, giardicida (Alonso \& Desmarchelier, 2005; Barboza et al., 2009). Tripogandra diuretica "Flor de Santa Lucía rosada". Usos: antihemorroidal, antirreumático, digestivo, diurético, sudorífico (Lahitte et al., 2004; Bacigalupo \& Hurrell, 2008). CRASSULACEAE: Crassula multicava "bálsamo tranquilo". Usos: cicatrizante y desinfectante, emético (Amorín \& Orfila, 1972; Pochettino et al., 1997). EUPHORBIACEAE: Euphorbia serpens "yerba meona". Usos: antinefrítico, antipirético, antiverrucoso, callicida, diurético, emenagogo, galactógeno, laxante (Pochettino et al., 1997; Lahitte et al., 2004; Barboza et al., 2006). Actividad biológica: antimicrobiano, diurético (Alonso \& Desmarchelier, 2005; Barboza et al., 2009). FABACEAE: Medicago polymorpha "trébol de carretilla". Usos: adelgazante (Lahitte \& Hurrell, 1996). Melilotus indicus "trébol de olor". Hoja compuesta peciolada, pinnado-trifoliolada. Usos: anticoagulante, diurético (Martínez Crovetto, 1981a; Lahitte \& Hurrell, 1996; Barboza et al., 2006); precaución: planta con principios tóxicos. Trifolium repens "trébol blanco". Usos: antirreumático, antisifilítico, depurativo, lavajes externos para curar llagas y granos, tónico (Ulibarri et al., 2002; Hurrell et al., 2011a, b). Actividad biológica: antiinflamatorio, antiulceroso, estrógeno (Barboza et al., 2006). FUMARIACEAE: Fumaria capreolata "flor de pajarito". Usos: depurativo, contra dermatosis 
y exantema, hipotensor, laxante, sedativo, tónico (Luján et al., 2004; Hurrell et al., 2011a). Debe usarse con precaución ya que posee alcaloides. GERANIACEAE: Geranium molle "alfilerillo". Usos: antiartrítico, antihidrópico, antipirético, astringente, galactófugo, hepático, vulnerario (Lahitte et al., 2004). IRIDACEAE: Sisyrinchium chilense ssp. chilense "canchalagua". Usos: antirreumático, carminativo, contra aftas, depurativo, digestivo, hepático, pectoral, tónico (Lahitte et al., 2004; Roitman \& Hurrell, 2009).

LAMIACEAE: Cantinoa mutabilis "salvia morada". Usos: antiespasmódico, antihelmíntico, antiodontálgico, antipirético, antirreumático, carminativo, desinfectante digestivo, diurético, estomacal (Martínez Crovetto, 1981a; Lahitte et al., 2004; Rondina et al., 2008). Actividad biológica: antiulcerante (Barboza et al., 2009). Mentha $\boldsymbol{x}$ piperita "menta". Usos: adelgazante, antiinflamatorio, antiespasmódico, antihelmíntico, antiséptico, aperitivo, carminativo, digestivo, estimulante estomacal, hipotensor, refrescante, (Pochettino et al., 1997; Mandrile, 2003; Barboza et al., 2006; Rondina et al., 2008; Hernández et al., 2010; Hurrell et al., 2011a). Actividad biológica: antibacteriano, antifúngico, carminativo, citotóxico, descongestivo, vasodilatador (Barboza et al., 2006). Ocimum basilicum "albahaca". Usos: antiespasmódico, antihelmíntico, antiinflamatorio, antiséptico, estomacal (Martínez Crovetto, 1981b; Pochettino et al., 1997; Sánchez Govín et al., 2000). Origanum vulgare L. "orégano". Usos: digestivo, estomacal, pectoral (Martínez Crovetto, 1981b; Pochettino et al., 1997; Hernández et al., 2013). Rosmarinus officinalis L. "romero". Usos: antiartrítico, antigripal, antirreumático, cardiotónico, contra los resfríos, digestivo, estomacal, hepático (Martínez Crovetto, 1981b; Pochettino et al., 1997; Hernández et al., 2010). Salvia officinalis "salvia blanca". Usos: digestivo, hepático (Hernández et al., 2009, 2010, 2013). MALVACEAE: Malva parviflora "malva verde". Usos: analgésico, antifúngico, emoliente, laxante, pectoral (Martínez Crovetto, 1981b; Pochettino et al., 1997; Rondina et al., 2008, 2010). Modiola caroliniana "sanalotodo". Usos: analgésico, depurativo, emoliente, en aplicación externa fomentos en heridas y granos, refrescante (Pochettino et al., 1997; Orfila \& D’Alfonso, 1999; Hurrell et al., 2006; Rondina et al., 2008). Actividad biológica: hipotensor (Barboza et al., 2009). Sida rhombifolia "escoba dura". Usos: antilítico, digestivo, para el dolor de picaduras de abejas y avispas, purgante (Lahitte et al., 2004; Hernández et al., 2010). Actividad biológica: anticonceptivo, antiespasmódico, antiinflamatorio, antimicrobiano, citotóxico, energizante (Alonso \& Desmarchelier, 2005; Barboza et al., 2009). ONAGRACEAE: Oenothera affinis "suspiros". Usos: analgésico en odontalgia, antiséptico, vulnerario (Lahitte et al., 2004; Barboza et al., 2006, 2009; Rondina et al., 2008). OXALIDACEAE: Oxalis articulata ssp. articulata "vinagrillo". Usos: antiescorbútico, antipirético (Lahitte et al., 2004; Barboza et al., 2009). PHYLLANTHACEAE: Phyllanthus niruri "rompe piedra". Usos: antidiabético, antilítico, antineurítico, astringente, contra la hidropesía y gota, diurético, (Martínez Crovetto, 1981a; Lahitte et al., 2004; Hurrell et al., 2007; Hernández et al., 2009). Actividad biológica: analgésico, antibacteriano, antihipertensivo, antilítico, antiviral, contra carcinoma ascítico, diurético, hepatoprotector, hipoglucemiante, hipotensor, relajante muscular (Alonso \& Desmarchelier, 2005; Barboza et al., 2009). PLANTAGINACEAE: Plantago lanceolada "siete venas". Usos: analgésico, antibiótico, antiinflamatorio, antiofídico, antiulceroso, aporte de vitamina C, astringente, contra irritación de los ojos por el sol, depurativo, digestivo, diurético, estomacal, hepático, pectoral, para picaduras de insectos y quemaduras (Kutschker et al., 2007; Rondina et al., 2008). Actividad biológica: antimicrobiano, bacteriostático (Barboza et al., 2006). P. major "llantén”. Usos: analgésico, antiácido, antidiarreico, antiinflamatorio, antipruritoso, antirreumático, antiséptico, astringente, digestivo (Kutschker et al., 2007; Hernández et al., 2010, 2013; Hurrell et al., 2011a; dos Santos et al., 2012). Actividad biológica: antibacteriano, antidiarreico, antifúngico, antiinflamatorio, antiparasitario, antiulceroso gástrico, citotóxico (Barboza et al., 2006). P. tomentosa ssp. tomentosa "llantén". Usos: analgésico, antihemorroidal, antiinflamatorio, antioftálmico, curar aftas y llagas bucales, depurativo, emoliente, pectoral (Martínez Crovetto, 1964, 1981a; Barboza et al., 2006, 2009). POACEAE: Cymbopogon citratus "pasto limón". Usos: antidiarreico, antiespasmódico, antiinflamatorio, antimicrobiano, antipirético, antitusivo, carminativo, digestivo, expectorante, hipotensor, sedativo (Hernández et al., 2010, 2013; Hurrell et al., 2011a; Bohm et al., 2012; Almeida et al., 2013). PORTULACACEAE: Portulaca oleracea "verdolaga". Usos: analgésico renal, antidiarreico, antiescorbútico, antihelmíntico, antinefrítico, antihemorrágico nasal, ayuda a la dentición, bronquiolítico, depurativo, diurético, emoliente, expectorante, fasciolicida, hepático, oftálmico, 
vulnerario (Hieronymus, 1882; Martínez Crovetto, 1981a; Lahitte \& Hurrell, 1996; Romero Jiménez et al., 2002; Rondina et al., 2008). Actividad biológica: antibacteriano, antiinflamatorio, antiulceroso (Barboza et al., 2006; Figueroa et al., 2011). SCROPHULARIACEAE: Verbascum virgatum "verbasco". Usos: antidiarreico, antiulceroso, emoliente, para curar sabañones (Hieronymus, 1882; Lahitte \& Hurrell, 1996; Lahitte et al., 2004). SOLANACEAE: Physalis viscosa "camambú". Usos: analgésico, antipirético, antitusivo, contra escoriaciones de la piel, digestivo, refrescante (Lahitte \& Hurrell, 1996; Lahitte et al., 2004; Barboza et al., 2006; Rondina et al., 2008). Actividad biológica: antibacterial (Barboza et al., 2009). Salpichroa origanifolia "huevito de gallo". Usos: fortalecedor capilar, fomentos para granos, narcótico, sedativo (Pochettino et al., 1997; Barboza et al., 2006). Actividad biológica: antibacteriano, antiinflamatorio (Barboza et al., 2009). Solanum sisymbriifolium "espina colorada". Usos: analgésico, antinefrítico, dermopático, hepático (Pochettino et al., 1997; Barboza et al., 2006). Planta peligrosa por la presencia de alcaloides. Actividad biológica: ansiolítico-sedante, antiinflamatorio, antimicrobiano, antitérmico, cardiotónico, contra melanomas, repelente de insectos (Alonso \& Desmarchelier, 2005). URTICACEAE: Parietaria debilis "parietaria". Usos: antipirético, contra granos y acné, diurético, lavado de llagas de viruela (Hieronymus, 1882; Lahitte \& Hurrell, 1996; Hurrell et al., 2007). Urtica urens "ortiga". Usos: analgésico, anticonceptivo, antidermatósico, antiinflamatorio, antilítico, antiparasitario, antiseborreico, diurético, fortificante capilar, rubefaciente (Pochettino et al., 1997; Barboza et al., 2006; Kutschker et al., 2007; Marrasini et al., 2010; Hurrell et al., 2011a). VERBENACEAE: Phyla nodiflora var. minor "yerba del mosquito". Usos: digestivo, hepático (Martínez Crovetto, 1981a; Lahitte et al., 2004; Hurrell et al., 2006). Actividad biológica: antimicrobiano (Barboza et al., 2009). Verbena bonariensis var. bonariensis "verbena". Usos: antidiabético, antiespasmódico, antigangrenoso (Tousarkissian, 1980; Martínez Crovetto, 1981a; Lahitte et al., 2004; Hernández et al., 2009). Actividad biológica: hipoglicémico (Barboza et al., 2009). Verbena litoralis var. litoralis "verbena". Usos: anticefalálgico, antipirético, digestivo, emenagogo, hepático (Martínez Crovetto, 1981b; Barboza et al., 2006; Rondina et al., 2008). Actividad biológica: antidiarreico, antimicrobiano, antioxidante, antitérmico, hipotensor, laxante, proctosigmoiditis (Alonso \& Desmarchelier, 2005; Barboza et al., 2009). Verbena montevidensis "verbena". Usos: como las restantes especies de Verbena (Lahitte \& Hurrell, 1996). 
